\title{
ISOPERIMETRY OF WAISTS AND CONCENTRATION OF MAPS
}

\author{
Misha GROMOV
}

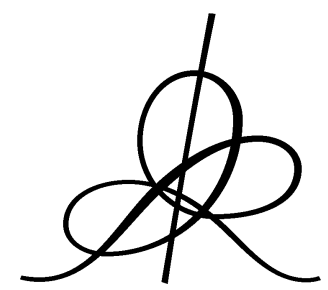

Institut des Hautes Études Scientifiques

35 , route de Chartres

91440 - Bures-sur-Yvette (France)

Février 2002

IHES/M/02/04 


\title{
Isoperimetry of waists and concentration of maps
}

\author{
Misha GROMOV
}

July 17,2002

\section{Waist of the sphere theorem}

Let $f: S^{n} \rightarrow \mathbb{R}^{k}$ be a continuous map where $S^{n}$ is the unit $n$-sphere. Then there exists a point $z \in \mathbb{R}^{k}$ such that the spherical $n$-volumes of the $\varepsilon$-neighbours of the level $Y_{z}=f^{-1}(z) \subset S^{n}$, denoted $Y_{z}+\varepsilon \subset S^{n}$, satisfy

$$
\operatorname{Vol}\left(Y_{z}+\varepsilon\right) \geq \operatorname{Vol}\left(S^{n-k}+\varepsilon\right) \quad(\star)_{S^{n}}
$$

for all $\varepsilon>0$, where $S^{n-k} \subset S^{n}$ denotes an equatorial $(n-k)$-sphere.

This is proven in 5.8.

Remarks. (a) If $k=n$, and card $f^{-1}(z) \leq 2, z \in \mathbb{R}^{n}$, then $(\star)_{S^{n}}$ applied to $\varepsilon=\pi / 2$ amounts to the Borsuk-Ulam theorem: some level $f^{-1}(z)$ of $f: S^{n} \rightarrow \mathbb{R}^{k}$ contains a pair of opposite points. Non-surprizingly, our argument in the general case depends on $\mathbb{Z}_{2}$-cohomological considerations.

(b) If $k=1$ one may take the Levy mean of $f$ for $z \in \mathbb{R}$, where the level $f^{-1}(z) \subset S^{n}$ divides the sphere into equal halves (i.e. where $\operatorname{Vol}\left(f^{-1}(-\infty, z]\right)$ and $\operatorname{Vol}\left(f^{-1}[z, \infty)\right)$ are both $\left.\geq \frac{1}{2} \operatorname{Vol}\left(S^{n}\right)\right)$. Then $(\star)_{S^{N}}$ follows from the spherical isoperimetric inequality (see 9.2.B).

(c) The inequality $(\star)_{S^{n}}$ for $\varepsilon \rightarrow 0$ shows that the Minkowski $m$-volume of $f^{-1}(z)$ for $m=n-k$ is $\geq$ than that of the equatorial sphere $S^{m} \subset S^{n}$. Yet, it remains unclear if some level of $f$ has the Hausdorff measure $\geq \operatorname{Vol}_{m}\left(S^{m}\right)$. (If $f$ is a generic smooth map, a level with Haumes $m \geq \operatorname{Vol}_{m}\left(S^{m}\right)$ is delivered by the Almgren-Morse theory, see [Gro $]_{\text {FRM. }}$ )

\section{Waists of $m m$-spaces}

An $m m$ space $X=(X$, dist, $\mu)$ is a metric space with a Borel measure $\mu$ on it. Customary one deals with Polish spaces, where $X$ is a dist-complete 
separable space. Our $X$ 's, for most part, are Riemannian manifolds, where $\mu$ may or may be not equal the corresponding Riemannian measure $d x$ of $\left(X\right.$, dist $\left._{\text {Riem }}\right)$. One says that $\mu$ is normalized or it is a probability measure if $\mu(X)=1$. In this case $X$ is called a $p m$ space.

We extend the $m m$ framework by allowing $X=(X$, dist, $\mu)$, where $\operatorname{dist}\left(x, x^{\prime}\right)$ becomes infinite on some pairs $\left(x, x^{\prime}\right) \in X \times X$. A basic example is the countable Cartesian power $X^{\infty}$ of a compact pm space $X$, where the Cartesian (i.e. Pythagorian) metric dist ${ }^{\infty}$ is infinite for almost all $\left(x, x^{\prime}\right) \in X^{\infty} \times X^{\infty}$. Yet $\operatorname{dist}^{\infty}\left(Y, Y^{\prime}\right)<\infty$ for measurable subsets $Y, Y^{\prime}$ of positive $\mu^{\infty}$-measure under rather mild assumptions on $(X=X$, dist, $\mu)$, e.g. for (connected!) Riemannian $X$ with normalized Riemannian measures (compare "concentrated spaces" in $[\mathrm{Gro}]_{\mathrm{SQ}}$ ).

Given a family $Z$ of subsets $Y=Y_{z} \subset X=(X$, dist, $\mu), z \in Z$, and a function $w(\varepsilon), \varepsilon>0$, we write

$$
\operatorname{wst}\left(X,\left\{Y_{z}\right\}, \varepsilon\right) \geq w(\varepsilon)
$$

if $\exists z$, s.t.

$$
\mu\left(Y_{z}+\varepsilon\right) \geq w(\varepsilon) \text { for all } \varepsilon>0,
$$

where $Y+\varepsilon$ denotes the $\varepsilon$-neighbourhood: $\{x \in X \mid \operatorname{dist}(x, Y) \leq \varepsilon\}$.

Given a topological space $Z$, write

$$
\operatorname{wst}(X \rightarrow Z, \varepsilon) \geq w(\varepsilon),
$$

if $\exists z \in Z$, such that for every continuous map $f: X \rightarrow Z$ there exists $z \in Z$, where the level $Y_{z}=f^{-1}(z) \subset X$ satisfies

$$
\mu\left(Y_{z}+\varepsilon\right) \geq w(\varepsilon)
$$

for all $\varepsilon>0$.

If one is concerned with an individual $\varepsilon=\varepsilon_{0} \geq 0$, one writes $\operatorname{wst}\left(X,\{Y\}, \varepsilon=\varepsilon_{0}\right) \geq w_{0}$ if $\mu\left(Y_{z}+\varepsilon_{0}\right) \geq w_{0}$ for some $z \in Z$. A particular value is $\varepsilon_{0}=0$, where one arrives at the Minkowski volume (measure) of codimension $k$, that is $c_{k} \liminf _{\varepsilon \rightarrow 0} \varepsilon^{-k} \mu\left(Y_{z}+\varepsilon\right)$, where $c_{k}=\left(\operatorname{Vol} B_{k}\right)^{-1}$ for the unit ball $B_{k} \subset \mathbb{R}^{k}$. The supremum of these over $z \in Z$ is denoted by Miwst $_{-k}\left(X,\left\{Y_{z}\right\}\right)$ and Miwst $_{-k}(X \rightarrow Z)$ is understood accordingly.

If $Z=X$ and $Y_{x}=\{x\} \subset X$, (or, speaking of $X \rightarrow Z$, we have a so large $Z$ that $X$ can be embedded into $Z$ ), then a lower bound on the waist amounts to finding a point $x_{0}$ with the balls $B\left(x_{0}, \varepsilon\right) \subset X$ of large measures. We set

$$
\text { wst }_{x_{0}}(X, \varepsilon)=\mu\left(B\left(x_{0}, \varepsilon\right)\right)
$$


and write wst. $(X, \varepsilon)$ for wst $_{x_{0}}(X, \varepsilon)$ if there is a specified non-ambiguous (choice of a distinguished) point $x_{0} \in X$. Sometimes, wst $_{x_{0}}(X, \varepsilon) \geq \operatorname{wst}_{x}(X, \varepsilon), x \in X$, simultaneously for all $\varepsilon>0$, as it happens, for instance, for the Gaussian spaces (see below), and then wst• refers to wst $_{x_{0}}$ for this $x_{0} \in X$.

\section{Gaussian spaces and log-concavity}

Let

$$
\mathrm{Ga}=(\mathbb{R}, g(x) d x)
$$

for

$$
g(x)=(2 \pi)^{-\frac{1}{2}} \exp -\frac{x^{2}}{2}
$$

and

$$
\mathrm{Ga}^{n}=\left(\mathbb{R}^{n},\left(g^{n}=(2 \pi)^{-\frac{n}{2}} \exp -\frac{\|x\|^{2}}{2}\right) d x\right) .
$$

A smooth positive function $\varphi$ on a convex subset $X$ in $\mathbb{R}^{n}$ and the corresponding measure $\varphi(x) d x$ are called $\lambda$-log-concave, if the second derivative $\partial^{2} \log \varphi(x)$ is $\leq-\lambda$ for all unit tangent vectors $\partial$ in $X$. For example $g(x) d x$ is log-concave that means $\lambda$-log-concave for $\lambda=1$.

\subsection{Log-waist theorem (see 5.7)}

Let $X$ be a convex subset in $\mathbb{R}^{n}$ with a log-concave probability measure. Then

$$
\begin{aligned}
& \operatorname{wst}\left(X \rightarrow \mathbb{R}^{k}, \varepsilon\right) \geq \operatorname{wst}_{\bullet}\left(\mathrm{Ga}^{k}, \varepsilon\right)=\int_{B(0, \varepsilon)} g^{k}(x) d x \\
& =\int_{0}^{\varepsilon}\left(x^{k-1} \exp -\frac{x^{2}}{2}\right) d x / \int_{0}^{\infty}\left(x^{k-1} \exp -\frac{x^{2}}{2}\right) d x .
\end{aligned}
$$

The inequality $(\star)_{\log }$ is sharp: for example, the equality is achieved for linear maps $\mathrm{Ga}^{n} \rightarrow \mathbb{R}^{k}$.

3.1.A Question. Let dist and $\mu$ be $O(n)$-invariant metric and measure in $\mathbb{R}^{n}$. Under what condition(s) is the waist inequality extremized by the $(n-k)$-plane through the origin? This is unknown (except for the case $k=1$ ), for instance, for the Euclidean measure restricted to the unit ball $B(0,1) \subset \mathbb{R}^{n}$ with the Euclidean metric. 


\subsection{Waists of powers}

Let $X$ be a compact connected Riemannian manifold with a probability measure $\mu=\varphi(x) d x$, where the function $\varphi: X \rightarrow \mathbb{R}_{+}$is continuous and $>0$ everywhere on $X$. Then there exists $\underline{\lambda}=\underline{\lambda}(X)>0$, such that all Cartesian powers $X^{N}, N=1,2, \ldots, \infty$, have

$$
\operatorname{wst}\left(X^{N} \rightarrow \mathbb{R}^{k}, \varepsilon\right) \geq \operatorname{wst}_{\bullet}\left(\mathrm{Ga}^{k}, \underline{\lambda} \varepsilon\right)
$$

for all $k=0,1, \ldots$ (where the continuity of $f: X^{\infty} \rightarrow \mathbb{R}^{k}$ is understood for the infinite product topology).

Proof. One sees easily that $X$ can be dominated by the space $\underline{\lambda}^{-1} \mathrm{Ga}^{n}$ for each $n \geq \operatorname{dim} X$ and $\underline{\lambda}=\underline{\lambda}_{n}=\underline{\lambda}(X$, dist, $\mu, n)\left(\lambda_{n}\right.$ monotone decrease in $n$ ), where

$$
\underline{\lambda}^{-1} X \stackrel{\text { def }}{=}\left(X, \underline{\lambda}^{-1} \text { dist }, \mu\right)
$$

and accordingly,

$$
\begin{gathered}
\operatorname{wst}\left(X^{N} \rightarrow \mathbb{R}^{k}, \varepsilon\right) \geq \operatorname{wst}\left(\left(z^{-1} \mathrm{Ga}^{n}\right)^{N} \rightarrow \mathbb{R}^{k}, \varepsilon\right)= \\
\text { wst. }_{\bullet}\left(\mathrm{Ga}^{k}, \underline{\lambda} \varepsilon\right)=\int_{0}^{\underline{\lambda} \varepsilon}\left(x^{k-1} \exp -\frac{x^{2}}{2}\right) d x / \int_{0}^{\infty}\left(x^{k-1} \exp -\frac{x^{2}}{2}\right) d x .
\end{gathered}
$$

Domination, written $X \prec X^{\prime}$ between $m m$ spaces $X=(X$, dist, $\mu)$ and $X^{\prime}=\left(X^{\prime}\right.$, dist $\left.^{\prime}, \mu^{\prime}\right)$, signifies the existence of a $\lambda$-Lipschitz map $\psi: X^{\prime} \rightarrow X$ that pushes forward $\mu^{\prime}$ to $\mu$, i.e. $\psi_{*}\left(\mu^{\prime}\right)=\mu$. The required domination of $X$ by some $\underline{\lambda}^{-1} \mathrm{Ga}^{n}$ is elementary (compare $\S 3 \frac{1}{2}$ in [G-L-P]) and then $(\star)_{N}$ follows from $(\star)_{\log }$ applied to $\mathrm{Ga}^{n N}=\left(\mathrm{Ga}^{n}\right)^{N}$, since

(a) the waist is obviously monotone for the domination ordering between $m m$ spaces.

(b) The domination order is consistant with the Cartesian products.

\section{3 $\mathbb{Z}_{2}$-waists and Gibbsian waists}

We shall introduce in 7.2 the notions of parametric $\mathbb{Z}_{2}$-waists, denoted $\mathbb{Z}_{2}$-wst $(X \rightarrow Z, \varepsilon)$, that minorize the corresponding ordinary waists, wst $\geq \mathbb{Z}_{2 \text {-wst }} \geq \mathbb{Z}_{2}$-pawst, i.e. $\mathbb{Z}_{2}$-wst $(\varepsilon) \geq w(\varepsilon) \Rightarrow \operatorname{wst}(\varepsilon) \geq w(\varepsilon)$ for all functions $w(\varepsilon)$ and similarly for pawst. Our proof of $(\star)_{S^{n}},(\star)_{\log }$ and $(\star)_{N}$ allows $\mathbb{Z}_{2}$-pawst, and hence, $\mathbb{Z}_{2}$-wst in these inequalities (see 7 ) where the advantage of the $\mathbb{Z}_{2}$-version is their stability under Cartesian products 
and other operations over $m m$ spaces. For example, we shall see in 7 (for certain fibrations as well as products) that

$\mathbb{Z}_{2^{-}}$pawst $\left(X \times X^{\prime} \rightarrow \mathbb{R}^{k}\right) \geq\left(\mathbb{Z}_{2^{-}} \operatorname{pawst}\left(X \rightarrow \mathbb{R}^{k}\right)\right)\left(\mathbb{Z}_{2^{-}} \operatorname{pawst}\left(X^{\prime} \rightarrow \mathbb{R}^{k}\right)\right)(\times)_{\ell_{\infty}}$

for all $k$ and all $m m$-spaces $X$ and $X^{\prime}$, where $X \times X^{\prime}$ is given the sup (or $\left.\ell_{\infty}\right)$ product metric

$$
\operatorname{dist}\left(\left(x_{1}, x_{1}^{\prime}\right),\left(x_{2}, x_{2}^{\prime}\right)\right)=\max \left(\operatorname{dist}_{X}\left(x_{1}, x_{2}\right), \operatorname{dist}_{X^{\prime}}\left(x_{1}^{\prime}, x_{2}^{\prime}\right)\right) .
$$

There is a similar inequality for the ordinary Cartesian $\left(\ell_{2}\right.$-Pythagorian $)$ products (and Riemannian fibrations) applicable not to the functions wst $(\varepsilon)$ but rather to their Gibbs-Laplace transforms. Namely, assigne, to each $Y \subset X$, the function

$$
\mathrm{Gi}(Y ; \beta)=\int_{X}\left(\exp -\beta \operatorname{dist}_{X}(x, Y)\right) \mu d x
$$

and modify the definition of the waists by substituting $\operatorname{Gi}\left(\left\{Y_{z}\right\}, \beta\right)$ and $\mathrm{Gi}\left(f^{-1}(z), \beta\right)$ in the place of $\mu\left(Y_{z}+\varepsilon\right)$ and $\mu\left(f^{-1}(z)+\varepsilon\right)$. The $(\star)$-inequalities obviously yield their Gi-version, and moreover, these Giwst's admit parametric $\mathbb{Z}_{2}$-refinements as well. The latter satisfy the Cartesian product inequality (see 7)

$$
\begin{gathered}
\mathbb{Z}_{2^{-}} \text {paGiwst }\left(X \times X^{\prime} \rightarrow \mathbb{R}^{k}, \beta\right) \geq \\
\left(\mathbb{Z}_{2^{-}} \operatorname{paGiwst}\left(X \rightarrow \mathbb{R}^{k}, \beta\right)\right) \times\left(\mathbb{Z}_{2^{-}} \operatorname{paGiwst}\left(X^{\prime} \rightarrow \mathbb{R}^{k}, \beta\right)\right) . \quad(\times)_{\mathrm{Gi}}
\end{gathered}
$$

\subsection{Remarks and examples}

One can combine $(\times)_{\text {Gi }}$ with the $\mathbb{Z}_{2}$-refined $(\star)_{S^{n}}$ and thus evaluate Giwst's of products of spheres. However, the resulting inequalities are far from being sharp. A better (still not sharp) result can be obtained with a domination of the half-sphere $S_{+}^{n}$ (with the normalized Riemannian-measure) by $\lambda_{n}^{-1} \mathrm{Ga}^{n}$, where $\lambda_{n}$ can be explicitly evaluated by looking at $O(n)$-invariant (dominating) maps $\left(\mathbb{R}^{n}, g^{n}(x) d x\right) \rightarrow S_{+}^{n}$, where the problem reduces to domination of the measure $(\sin t)^{n-1} d t$ by suitably normalized $t^{n-1}\left(\exp -\lambda_{n} t^{2}\right) d t$. Thus one can show that the products of the spheres $r_{i} S^{n_{i}}, i=1, \ldots, j$ of radii $r_{i}$ with normalized measures have the waists bounded by the infimum of the corresponding Gaussian waists. Namely, let $\underline{\lambda}=\min _{i=1, \ldots, j}\left(\lambda_{n_{i}} / r_{i}\right)$. Then the product $X=r_{1} S^{n_{1}} \times \cdots \times r_{j} S^{n_{j}}$ has

$$
\operatorname{wst}\left(X \rightarrow \mathbb{R}^{k}, \varepsilon\right) \geq \operatorname{wst}_{\bullet}\left(\underline{\lambda}^{-1} \mathrm{Ga}^{k}, \varepsilon\right) \quad(\times)_{S_{+}^{n}}
$$

for all $k=0,1, \ldots$ (see 7$)$. 


\subsection{Concentration and upper bounds on waists of $X^{N \rightarrow \infty}$}

If $\operatorname{wst}\left(X \rightarrow \mathbb{R}^{k}, \varepsilon\right) \geq w(\varepsilon)$ then, obviously, for every 1-Lipschitz map the push-forward measure $\mu_{*}=f_{*}(\mu)$ on $\mathbb{R}^{k}$ (for the measure $\mu$ on $X$ ) is $w$ concentrated at some point $z_{0} \in \mathbb{R}^{k}$ :

$$
\operatorname{wst}_{z_{0}}\left(\left(\mathbb{R}^{k}, \mu_{*}\right), \varepsilon\right) \geq w(\varepsilon) . \quad(*)_{\text {Lip }}
$$

Thus the $(\star)$-inequalities yield the corresponding $(*)$-inequalities for $\mu_{*}$.

One can use $(*)_{\text {Lip }}$ the other way around and bound $\operatorname{wst}\left(X^{\infty} \rightarrow \mathbb{R}^{k}\right)$ by wst. $\left(\bar{\lambda}^{-1} \mathrm{Ga}^{k}\right)$ for some $\bar{\lambda}=\bar{\lambda}(X)>0$ as follows.

Given a compact $p m$ space $X$, consider 1-Lipschitz functions $f: X^{N} \rightarrow$ $\mathbb{R}, \quad N=1,2, \ldots$, with $\int_{X^{N}} f(x) \mu^{N} d x=0$ and let $\Lambda=\Lambda(X)=$ $\sup _{f, N} \int_{X^{N}} f^{2}(x) \mu^{N} d x$ over all such $f$ and $N=1,2, \ldots$ Let $\bar{\lambda}=\bar{\lambda}(X)$ is defined by the relation

$$
\int_{-\infty}^{+\infty} \bar{\lambda}^{2} x^{2} g(x) d x=\Lambda
$$

Then

$$
\operatorname{wst}\left(X^{\infty} \rightarrow \mathbb{R}^{k}, \varepsilon\right) \leq \operatorname{wst}_{\bullet}\left(\bar{\lambda}^{-1} \mathrm{Ga}^{k}\right) \quad(\leq)_{\infty}
$$

for all $k=1,2, \ldots$ where this inequality must be understood as follows: for every pair of numbers $0<\varepsilon_{0}<\varepsilon_{1}<\infty$ and each $\delta>0$ there exists a continuous (in fact 1-Lipschitz) map $F: X^{N} \rightarrow \mathbb{R}^{k}$ for all sufficiently large $N \geq N\left(\varepsilon_{0}, \varepsilon_{1}, \delta\right)$, such that all levels $Y_{z}=F^{-1}(z), z \in \mathbb{R}^{k}$ satisfy

$$
\mu^{N}\left(Y_{z}+\varepsilon\right) \leq \mu_{\mathrm{Ga}}(B(0, \bar{\lambda} \varepsilon))-\delta
$$

for all $\varepsilon \in\left[\varepsilon_{0}, \varepsilon_{1}\right]$.

Proof. Take a 1-Lipschitz map $f: X^{N_{0}} \rightarrow \mathbb{R}$ with $\int f^{2}$ approaching $\Lambda$, and let $f_{N_{1}}=\frac{1}{\sqrt{N_{1}}} \sum_{i=1}^{N_{1}} f\left(x_{i}\right), x_{i} \in X^{N_{0}}$, on $X^{N_{1} N_{0}}$. The distribution of $f_{N_{1}}$ approaches $\bar{\lambda}^{-1} g(t) d t$ by the central limit theorem and $F=\left(f_{N_{1}}\right)^{k}$ : $X^{N_{1} N_{0}} \rightarrow \mathbb{R}^{k}$ does the job for $N_{0}, N_{1} \rightarrow \infty$.

Question. Obviously, $\bar{\lambda}(X) \geq \underline{\lambda}(X)$, but it is unclear what is a possible gap between the two numbers. 


\section{Concavity classes and convex derivation of mea- sures}

A concavity class $\mathcal{C}$ of measures on a polish space $X$ is, by definition, a cone in the space of Borel measures on $X$ stable under infima of measures: for every family of measures $\mu_{i} \in \mathcal{C}, i \in I$, there exists a unique measure $\mu^{\vee} \in \mathcal{C}$, such that

$$
\mu^{\vee} \leq \mu_{i}, i \leq I
$$

and $\mu^{\vee} \geq \mu$ for all measures $\mu$ satisfying $\mu \leq \mu_{i}, i \in I$. In our applications concavity classes are of the form $\{\varphi(x) d x\}$ for some background measure $d x$ on $X$, where the (density) functions $\varphi(x)$ are distinguished by some (local) concavity condition.

Examples. (a) An $x$-concave (or just concave) measure on $\mathbb{R}^{n}$ is supported on a convex subset $S$ (of possibly positive codimension) in $\mathbb{R}^{n}$ and is of the form $\varphi(s) d s$ for a concave function $\varphi(s)$ and the Lebesgue measure $d s$ on $S$.

(b) A smooth $\lambda$-log-concave $\mu$ on $S$ is a one where $\varphi(s)$ is $\lambda$-log-concave, i.e. $\partial^{2} \log \varphi(s) \leq-\lambda$. Then one defines (non-smooth) $\lambda$-log-concavity by taking infima of the smooth $\lambda$-log-concave $\varphi$ 's. Intuitively, these are "more concave" than $\exp -\frac{\lambda}{2}\|x\|^{2}$.

(c) $x^{k}$-concavity refers to densities $\varphi$ that are "more concave" than the function $x^{k}$ on $\mathbb{R}_{+}$, i.e. $\varphi^{\frac{1}{k}}(x)$ is a concave function.

(d) sin-concavity: these $\varphi$ are more concave than $\sin (x), x \in[0, \pi]$; if $\varphi$ is smooth this is expressed by the inequality $\partial^{2} \varphi \leq-\varphi$ and, in general, one takes infima of such $\varphi$ 's.

(e) $\sin ^{k}$-concavity: this means $\varphi^{\frac{1}{k}}$ is sin-concave.

The notion of $\sin ^{k}$-concavity is useful for convex subsets $S$ in $S^{n}$, where it refers to such concavity (of the density function $\varphi$ of the measure in question) on each geodesic segment in $S$. (Similarly, one defines various classes of concave function and measures on general Riemannian and some Finsler manifolds.)

\subsection{Products of concavity classes}

Given a (concavity) class $\mathcal{C}_{0}$ of measures $\mu$ on $X$ and a (concavity) class $\Phi$ of positive functions $\varphi$ one defines $\mathcal{C}=\Phi \cdot \mathcal{C}_{0}$ as the minimal concavity class of 
measures containing the products $\varphi \mu$ for all $\varphi \in \Phi$ and $\mu \in \mathcal{C}$. This is well defined as the intersection $\mathcal{C}=\bigcap_{i \in I} \mathcal{C}_{i}$ of concavity classes $\mathcal{C}_{i}$ is a concavity class.

Examples. (a) Given a class $\mathcal{C}_{0}$ of measures on (convex subsets in) $\mathbb{R}^{n}$ one defines $\mathcal{C}=x^{k} \mathcal{C}_{0}$ as $\Phi_{x^{k}} \cdot \mathcal{C}_{0}$ for the class $\Phi_{x^{k}}$ of $x^{k}$-concave functions. One checks elementary that this transforms $x^{\ell}$-concave measures to $x^{k+\ell}$-concave ones and that the class of $\lambda$-log-concave measures goes into itself.

(b) Multiplication by $(\sin )^{k}$-concave functions transforms the class $\mathcal{C}_{\sin ^{\ell}}$ of $\sin ^{\ell}$-concave measures to $\mathcal{C}_{\sin ^{\ell+k}}$.

\subsection{Derived classes}

Given a class $\mathcal{C}$ of measures $\mu$ on $X=S^{n}$ or $\mathbb{R}^{n}$ (or on an arbitrary Finsler space for this matter) one restricts of these $\mu$ to open convex subsets $U$, normalizes them by taking $\mu_{\text {nor }} \mid U=(\mu \mid U) / \mu(U)$ and defines $\mathcal{C}^{\prime}$ as the set of the weak limits of $\mu_{\text {nor }} \mid U$ for all $\mu \in \mathcal{C}$ and $U \subset X$. The measures $\mu^{\prime} \subset \mathcal{C}^{\prime}$ are called convexly derived from measures $\mu$ in $\mathcal{C}$ and they acquire new concavity property when the implied sequences of convex subsets $U_{i}$ degenerate, i.e. Hausdorff converge to convex subsets $S \subset X$ of positive condimention $k$. The Brunn-Minkowski theorem claims, for example, that the measures on such $S$ derived from $x^{\ell}$-convex measures in $\mathbb{R}^{n}$ are $x^{k+\ell}$-convex and $\lambda$ log-concave measures remain $\lambda$-log-concave, while in $S^{n}$ this codimension $k$-degeneration brings $(\sin )^{\ell}$-concave measures to $(\sin )^{\ell+k}$-concave ones. In general, under mild (if any) assumption on $\mathcal{C}$, it transforms to $x^{k} \mathcal{C}$ for the codimension $k$ degeneration in $\mathbb{R}^{n}$ and to $\sin ^{k} \mathcal{C}$ in $S^{n}$ (compare [Gro-Mil]).

Remark. The latter generalizes to arbitrary Riemannian manifolds $X$ as follows. Let a family of geodesicly convex open subsets $U$ Hausdorff converges to some $S$ with $\operatorname{codim} S=k$ and the normalized Riemannian measures $d x$ in $U$ 's weakly converge to some measure $\mu$ on $S$ with the density function $\varphi(s)$ with respect to the Riemannian measure $d s$ on $S$. Take a unit tangent vector $\partial$ to $S$ at some point $s \in S$, an orthonormal frame $\nu_{1}, \ldots, \nu_{k}$ of normal vectors and let $R(S, \partial)$ denotes the sum of the sectional curvatures on the bi-vectors $\partial \wedge \nu_{i}, i=1, \ldots, k$. Then the concavity of the restriction of $\varphi$ to each geodesic segment $\sigma$ in $S$ is controlled by the lower bound on $R(S, \partial)$ for the unit tangent vectors $\partial$ to $\sigma$ : if $R(S, \partial) \geq k\left(=\operatorname{Ricci} S^{k+1}\right)$ on $\sigma$ then $\varphi(\sigma)$ is $\sin ^{k}$-concave. This can be derived from the Paul Levy 
inequality for convex subsets in manifolds with Ricci $\geq k$ or can be proven, I guess, by a direct elementary argument.

Questions. (a) Let a geodesic segment $S$ without conjugate points in a Riemannian manifold $X$ be given. When can $S$ be approximated by open geodesicly convex subsets in $X$ ? (The same question for convex subsets $S$ of dimension $n-k>1$ seems relevant for symmetric spaces $X$.)

(b) What happens if instead of convex $U \subset S^{n}$ in the definition of convex derivation of measures one uses another class of open subsets of "bounded complexity", for example, semi-algebraic $U$ 's of degree $\leq d$ ?

\subsection{Convex partitions (compare [Gro-Mil])}

A measurable partition $\Pi$ of $S^{n}$ (or of a convex subset in $S^{n}$ ) into convex subsets $S=S_{\pi}, \pi \in \Pi$, is called convex if it equals the limit of consecutive refinements of finite partitions into convex subsets. Every probability measure $\mu$ on $S^{n}$, induces the canonical system of probability measures $\mu_{\pi}$ on almost all $S_{\pi}$ and these $\mu_{\pi}$ are convexly derived from $\mu$ for almost all $\pi \in \Pi$ (see [Gro-Mil]). Thus almost all $\mu_{\pi}$ are $\sin ^{k}$-concave for the Lebesgue measure $\mu=d s$, provided codim $S_{\pi} \geq k$ for all $\pi \in \Pi$ and these are 1-log-concave if $\mu$ is 1-log-concave, e.g. equals the Gaussian measure.

\subsection{Convex partition theorem}

Let $\mu$ be a Borel probability measure on $S^{n}$ and let $\mathcal{M}_{k}^{\prime}$ denote the space of weak limits $\mu^{\prime}$ of the restrictions $\left(\mu \mid U_{i}\right) / \mu\left(U_{i}\right)$ for all sequences of convex open $U_{i}$ 's that Hausdorff converge to compact convex subsets $S \subset S^{n}$ of codimension (exactly!) $k$. Clearly, $S \supset \operatorname{supp} \mu^{\prime}$ for all convex $S \subset S^{n}$, and, to save notations, we assume that $S=\operatorname{supp} \mu^{\prime}$ for all $\mu^{\prime} \in \mathcal{M}_{k}^{\prime}$.

Let $c_{\bullet}^{k}: \mathcal{M}_{k}^{\prime} \rightarrow S^{n}$ be a continuous ("center") map, such that $c_{\bullet}^{k}\left(\mu^{\prime}\right) \in$ $S=\operatorname{supp} \mu^{\prime}$ for all $\mu^{\prime}$ (e.g. the center of mass of $\mu^{\prime}$ ). The map $c_{\text {• }}^{k}$ does not, in general, continuously extend to the weak closure of $\mathcal{M}_{k}^{\prime}$ : when a sequence $\left(S_{i}^{\prime}, \mu_{i}^{\prime}\right)$ converges to $\left(S^{\prime \prime}, \mu^{\prime \prime}\right)$ with $\operatorname{codim} S^{\prime \prime} \geq k+1$ the sequence $c_{\bullet}^{k}\left(\mu_{i}^{\prime}\right) \in S_{i}^{\prime}$ does not have to converge. In such a case we take the limit points of all these (sub)sequences and denote by $c_{\circ}\left(\mu^{\prime \prime}\right) \subset S^{\prime \prime}$ the (compact) subset consisting of all such limits. If $\Pi$ is a convex partition of $S^{n}$ into $S_{\pi}$ 's with codim $S_{\pi} \geq k$, we denote by $C_{\pi} \subset S_{\pi}$ the ("center") image of the (set valued) map sending each $S_{\pi}$ with codimension $=k$ to $c_{\bullet}^{k}\left(\mu_{\pi}\right)$ and if $\operatorname{codim} S_{\pi} \geq k+1$ to $c_{0}\left(\mu_{\pi}\right)$, where $\mu_{\pi}$ denote the canonical system of measures associated to $\pi$. As we know these $\mu_{\pi}$ are convexly derived from 
$\mu$ for almost all $\pi$ and thus the ("central") subset $C_{\pi}$ is well defined in $S_{\pi}$ for almost all $\pi$.

4.4.A (see 6). Let $f: S^{n} \rightarrow \mathbb{R}^{k}$ be a continuous map. Then there exist a value $z \in \mathbb{R}^{k}$ and a convex partition $\Pi$ of $S^{n}$ into $S_{\pi}$ 's of codimension $\geq k$, such that the level $Y_{z}=f^{-1}(z) \subset S^{n}$ meets (the "center") $C_{\pi}$ for almost all $\pi \in \Pi$.

The spherical and the log-concave waist theorem easily follow from 4.4.A. The map $c_{\bullet}^{k}: \mu_{\pi} \mapsto s \in S_{\pi}$ one employs here is the assignement of the maximum point of the density function $\varphi(s)$ of $\mu_{\pi}$ on $S_{\pi}$. The $\sin ^{k}$ - and log-concavity respectively of $\varphi$ makes this $c_{\bullet}$ well defined and continuous in $\mu_{\pi}$. Since

$$
\mu\left(Y_{z}+\varepsilon\right)=\int_{\Pi} \mu_{\pi}\left(S_{\pi} \cap Y_{z}+\varepsilon\right) d \pi,
$$

(by the definition of the canonical system of measures), everything reduces to showing that the waists of almost all $\left(S_{\pi}, \mu_{\pi}\right)$ at $c_{\bullet}^{k}\left(\mu_{\pi}\right)$ are bounded from below by the normalized $\operatorname{Vol}\left(S^{n-k}, \varepsilon\right)$ and $\operatorname{wst}_{\bullet}(\mathrm{Ga}, \varepsilon)$ correspondingly. This trivially reduces to similar bounds for $\sin ^{k}$-concave and log-concave measures on $\mathbb{R}_{+}$with the maximum densities points at the origin, where the proof follows from the following

Elementary lemma. Let $\varphi(t), t \in\left[0, t_{0} \leq \infty\right)$ be a smooth positive monotone decreasing function where, $(\log \varphi)^{\prime \prime} \leq 0$, and, moreover, $(\log \varphi)^{\prime \prime}$ is monotone decreasing. Let $\lambda(\tau), \tau \leq \tau_{0}<t_{0}$ be a positive function and $\psi(t)=\inf _{0 \leq z \leq z_{0}} \lambda(\tau) \varphi(t+\tau)$. Then

$$
\int_{0}^{\varepsilon} \psi(t) d t / \int_{0}^{t-\tau_{0}} \psi(t) d t \geq \int_{0}^{\varepsilon} \varphi(t) d t / \int_{0}^{t_{0}} \varphi(t) d t .
$$

The proof is straightforward. In the $S^{n}$-case this applies to $\varphi(t)=\cos t$, $t \in[0, \pi / 2]$ and in the log-concave case to $\varphi(t)=\exp -t^{2}$.

Question. Does every closed subset $Y \subset S^{n}$ passing through the "centers" $C_{\pi}$ of all $S_{\pi}$ making a $k$-dimensional convex partition of $S^{n}$ necessarily has Haumes $_{n-k} Y \geq \operatorname{Vol} S^{n-k}$ ? (The transversal $(n-k)$-measure of the partition looks like a calibrating $(n-k)$-form with norms $\leq 1$ on $Y$ that integrates to $\geq \operatorname{Vol} S^{n-k}$.)

\section{Spaces of partitions}

A convex partition of depth $i$ is obtained by the following $i$-step process. 
Step 1. Divide $S^{n}$ into halves by an (co)oriented hyperplane i.e. oriented equatorial hypersphere $h \subset S^{n}$.

Step i. Divide each convex subset $S$ defined on the $(i-1)$-th step into two parts by an oriented hyperplane.

The resulting space $\mathcal{P}_{i}$ of partitions $\Pi$ of depth $i$ identifies with the Cartesian power $H^{2^{i}-1}$ where $H=H^{n}=\left(S^{n}\right)^{*}$ is the space of oriented hyperplanes $h \in S^{n}$; this $H$ is canonically homeomorphic to $S^{n}$, where each $k$ is determined by the unit normal vector $h^{\perp} \in S^{n}$. The $2^{i-1}$ hyperplanes making a point in $\mathcal{P}_{i}$ are organized into a rooted diadic tree $T_{i}$ of depth $i$ : the interior vertices $t \in T_{i}$ have the outcoming degrees 2 . The leaves of $T_{i}$ mark the subsets $S_{\pi} \subset S$ making a partition.

The group $G_{i}$ of the automorphisms of $T_{i}$ naturally acts on $\mathcal{P}_{i}$ by permutations of the coordinates $h_{t} \in H t \in\left\{\right.$ interior vertices of $\left.T_{i}\right\}$ and central reflections of $H$ for $h \leftrightarrow-h$ where $h \mapsto-h$ indicates reversal of the (co)-orientation of the hyperplane $h$. Namely, if a vertex $t$ goes to $t^{\prime}$ by $g: T_{i} \rightarrow T_{i}$, then $h_{t}$ goes to $h_{t^{\prime}}$ or $-h_{t^{\prime}}$ depending on wether $g$ preserves or reverses the order of the two edges growing from $t_{j}$ here we assume that all vertices $t$ of a given level $j$ (i.e. of distance $j$ from the root of $T_{i}$ ) are naturally (lexicographically) ordered, while $G_{i}$ consists of combinatorial automorphisms of $T_{i}$ irrespectful of the order. Thus $\operatorname{card}\left(G_{i}\right)=2^{i}$ and it is generated by involutions $i_{t}$ that switch two branches growing from the vertex $t \in T_{i}$. There is a natural homomorphism $\rho_{i}: G_{i} \rightarrow G_{i-1} i=2,3, \ldots$, where $\operatorname{ker} \rho_{i} \subset G_{i}$ is canonically isomorphic to $\left(\mathbb{Z}_{2}\right)^{2^{i-1}}$.

The action of $G_{i}$ on $\mathcal{P}_{i}$ is (obviously) free and we set $\mathcal{Q}_{i}=\mathcal{P}_{i} / G_{i}$. The Cartesian projection $\mathcal{P}_{i} \rightarrow \mathcal{P}_{i-1}$ induces a fibration $\mathcal{Q}_{i} \rightarrow \mathcal{Q}_{i-1}, i=$ $2,3, \ldots$, where the fiber is the $2^{i-1}$-th Cartesian power of the projective space $P^{n}=H^{n} /\{+1,-1\}$. The fundamental group $\pi_{1}\left(\mathcal{Q}_{i-1}\right)=G_{i-1}$ acts on $\left(P^{n}\right)^{2^{i-1}}$ by permuting coordinates; these permutations naturally extend to isomorphism between the standard line bundles $\ell$ over projective spaces. Thus the Whitney power $(\ell)^{2^{i-1}}$ over $\left(P^{n}\right)^{2^{i-1}}$ designs a vector bundle $L_{i}^{\prime} \rightarrow$ $\mathcal{Q}_{i}$ whose restriction to each fiber equals $(\ell)^{2^{i-1}}$. Besides $L_{i}^{\prime}$, the manifold $\mathcal{Q}_{i}$ carries the bundles $L_{j}^{*}, j=1,2, \ldots, i-1$ induces from $L_{j}^{\prime}$ over $\mathcal{Q}$. The Whitney sum of all of them is called $L_{i} \rightarrow \mathcal{Q}_{i}$,

$$
L_{i}=L_{1}^{*} \oplus L_{2}^{*} \oplus \cdots \oplus L_{i-1}^{*} \oplus L_{i}^{\prime} .
$$

\section{$5.1 \quad$ Non-vanishing Lemma}

For each $m \leq n$, the top Stiefel-Whitney class of the $m$-th Whitney's power 
$L_{i}^{m}$ does not vanish

$$
w_{N}\left(L_{i}^{m}\right) \neq 0, \text { for } N=N_{m}=m\left(2^{i}-1\right) .
$$

Proof. Since $w_{N_{m}} \cup w_{N_{\ell}}=w_{N_{m+\ell}}$, it suffices to prove the lemma for $m=$ $n$. The class $w_{N_{n}}\left(\left(L_{i}^{\prime}\right)^{n}\right)$ restricts to the generator in $H^{N^{\prime}}\left(\left(P^{n}\right)^{2^{i-1}} ; \mathbb{Z}_{2}\right)=$ $\mathbb{Z}_{2}, N^{\prime}=n 2^{i-1}$ on each fiber of the fibration $\mathcal{Q}_{i} \rightarrow \mathcal{Q}_{i-1}$, while $w_{N_{n}}\left(L_{i-1}^{n}\right)$ may be assumed $\neq 0$ by induction. Hence

$$
w_{N_{n}}\left(L_{i}^{n}\right)=w_{N_{n}}\left(\left(L_{i}^{\prime}\right)^{n}\right) \cup w_{N_{n}}^{*}\left(L_{i-1}^{n}\right) \neq 0,
$$

where $w_{N_{n}}^{*}$ denotes the pull-back of $w_{N_{n}}^{*}$ under $\mathcal{Q}_{i} \rightarrow \mathcal{Q}_{i-1}$. Q.E.D.

Remark. The dual homology class $w_{N_{n}}^{\perp}\left(\left(L_{i}^{\prime}\right)^{n}\right) \in H_{N-N^{\prime}}\left(\mathcal{Q}_{i} ; \mathbb{Z}_{2}\right)$ can be realized by the section $\mathcal{Q}_{i-1} \rightarrow \mathcal{Q}_{i}$ corresponding to a diagonal point in $\left(P^{n}\right)^{2^{i-1}}$ that is fixed under the monodromy, while $\left(w_{N_{n}}^{*}\right)^{\perp} \in H_{*}^{N^{\prime}}\left(\mathcal{Q}_{i} ; \mathbb{Z}_{2}\right)$ can be represented by a fiber of $\mathcal{Q}_{i} \rightarrow \mathcal{Q}_{i-1}$. Thus the non-vanishing is seen geometrically as the two cycles transversally intersect at a single point.

\subsection{Vanishing corollary}

Every continuous section $\mathcal{Q}_{i} \rightarrow L_{i}^{m}, m \leq n$, vanishes at some point $q \in \mathcal{Q}_{i}$. Moreover, the zero set of a section supports a non-zero homology class in $H^{N_{m}}\left(\mathcal{Q}_{i} ; \mathbb{Z}_{2}\right)$.

Notice that for $i=1$ this amounts to the Borsuk-Ulam theorem.

\section{$5.3 \quad c_{\bullet}$-corollary}

Denote by $\mathcal{S}$ the space of all $n$-dimensional geodesically convex subsets $S \subset$ $S^{n}$, each contained in a hemisphere, with the Hausdorff topology and let $S \mapsto c_{\bullet}=c_{\bullet}(S) \in S^{n}$ be a continuous map $\mathcal{S} \rightarrow S^{n}$. Then for each continuous map $f: S^{n} \rightarrow \mathbb{R}^{k}, k=1,2, \ldots, n-1$, there exists a partition $\Pi \in \mathcal{P}_{i}$ of $S^{n}$, for each $i=1,2, \ldots$, such that

(i) All convex subsets $S_{\pi}$ making $\Pi$ have equal $n$-dimensional volumes $=\operatorname{Vol}_{n}\left(S^{n}\right) / 2^{i}$;

(ii)

$$
f\left(c_{\bullet}\left(S_{\pi}\right)\right)=f\left(c_{\bullet}\left(S_{\pi^{\prime}}\right)\right)
$$

for all $\pi, \pi^{\prime}$ in all $\Pi$. 
Proof. Take a (possibly empty) convex subset $S=S_{\pi} \subset S^{n}$ for some $\pi \in \mathcal{P}_{i}$ and let $S^{\perp}=S_{\pi^{\perp}}$ be the immediate neighbour subset, where $S$ and $S^{\perp}$ are two halves of a convex subset $S_{-}$divided by a hyperplane $h$ on the $i$-th (the last) step of the division process. Thus $S$ and $S^{\perp}$ correspond to two neighbour leaves in $T_{i}$, while $h$ represents the interior vertex in $T_{i}$ adjacent to the two leaves. Set

$$
v(h)=\operatorname{vol}_{n}(S)
$$

and

$$
\varphi(h)=v(h) f\left(s_{\bullet}(S)\right) .
$$

Observe, that $h$ is (co)oriented and thus there is a non-ambiguous choice between $S$ and $S^{\perp}$, say corresponding to the "left" leaf in the tree $T_{i}$.

Next, take a hyperplane $h_{-}$appearing on the $j$-th step of the division process for $j<i$ and let $T_{h_{-}} \subset T_{i}$ be the "left" subtree in $T_{i}$ issuing upstream from (the vertex corresponding to) $h$. Let $\Pi_{h_{-}} \subset T_{i}$ consist of the leaves of $T_{i}$ contained in $T_{h_{-}}$. Take all convex subsets $S_{\pi}$, corresponding to $\pi \in \Pi_{h_{-}}$ and $h_{\pi}$ the respective (final) hyperplanes. Set

$$
\begin{gathered}
v\left(h_{-}\right)=\sum_{\pi \in \Pi_{h_{-}}} v\left(h_{\pi}\right) \\
\varphi\left(h_{-}\right)=\sum_{\pi \in \Pi_{h_{-}}} \varphi\left(h_{\pi}\right) .
\end{gathered}
$$

Now, to each point $\pi \in \mathcal{P}_{n}$ given by $\left\{h_{t}\right\}$ for $t \in\left\{\right.$ interior vertices of $\left.T_{i}\right\}$, we assigne the vector $F(\pi)$ in $\left(\mathbb{R}^{n-k+1}\right)^{2^{i}-1}$ by the rule

$$
F:\left\{h_{t}\right\} \mapsto\left\{\left(v\left(h_{t}\right)-v\left(-h_{t}\right), \varphi\left(h_{t}\right)-\varphi\left(-h_{t}\right)\right)\right\} .
$$

The map $F: \mathcal{P}_{i}=\left(H^{n}\right)^{2^{i}-1} \rightarrow\left(\mathbb{R}^{k}\right)^{2^{i}-1}$ is equivariant for the action of $G_{i}$ on $\mathcal{P}_{i}$ as well as the similar action on $\left(\mathbb{R}^{k}\right)^{2^{i}-1}$ and it is, clearly, continuous. Thus $F$ defines a section of $L_{i} \rightarrow \mathcal{Q}_{i}$ and, hence has a zero point $\pi \in \mathcal{P}_{i}^{i}$ which satisfies (i) and (ii) by the definition of $F$. Q.E.D.

\subsection{Example}

Let $c_{\bullet}(S) \in S$ be the center of the maximal $n$-ball inside $S$. Then the above delivers a convex partition of $S^{n}$ into $2^{i}$ subsets of equal volume, where some level $f^{-1}(\sigma) \subset S^{n}$ meets the centers of all $S_{\pi}$. 


\subsection{On $i=\infty$}

By passing to the projective limit $\mathcal{Q}_{\infty}$ for $\cdots \mathcal{Q}_{i} \rightarrow \mathcal{Q}_{i-1} \rightarrow \cdots \rightarrow \mathcal{Q}_{1}$ one obtains a similar result for infinite partitions that are our eventual destinations but, for the purposes of the present paper, one can reduce everything one needs to $i<\infty$, by several standard (albeit artificial) regularization tricks. A problem which presents itself however, is the dimension bound on the convex subsets $S_{\pi} \subset S^{n}$ making the final infinite partition: we need $\operatorname{dim} S_{\pi} \in k$ for all (at least, almost all) $\pi$. This is achieved with the following.

\subsection{Generalization of the vanishing lemmas}

Suppose, in the context of 7 we are given equatorial spheres $H_{1}^{m} \subset H^{n}$, $H_{2}^{m} \subset H^{n}, \ldots, H_{i}^{m} \subset H^{n}$. If $m \geq k+1$, then there exists a partition $\pi \in \mathcal{P}_{i}$ satisfying (i) and (ii) of 5.3 where, moreover, all hyperplanes $h \in H^{n}$ of level $j$ are contained in $H_{j}^{m}$ for all $j=1, \ldots, i$.

Proof. Proceed as before with $\mathcal{P}_{i}=\mathcal{P}_{i}\left(S^{n}\right)$ replaced by $\mathcal{P}_{i}\left(S^{m}\right)$ in the non-vanishing lemma.

\section{$5.7 \quad c_{\bullet}$-Corollary}

There exists a convex partition $\Pi$ of $S^{n}$ into $S_{\pi}$ with $\operatorname{dim} S_{\pi} \leq k$ for all $\pi$, such that

$$
f\left(c_{\bullet}\left(S_{\pi}\right)\right)=f\left(c_{\bullet}\left(S_{\pi^{\prime}}\right)\right) \quad(+)_{\bullet}^{\infty}
$$

for all pairs $\pi, \pi^{\prime} \in \Pi$, where $c_{\bullet}\left(S_{\pi}\right)$ for $\operatorname{dim} S_{\pi}<n$ refers to some limit point of $c_{\bullet}\left(S_{\pi_{i}}\right)$ for $S_{\pi}=\bigcap_{i=1}^{\infty} S_{\pi_{i}}$, where $\pi_{i} \in \mathcal{P}_{i}$ are finite partitions converging to $S_{\pi}$.

Proof. To make $\operatorname{dim} S_{\pi} \leq k$, we take a sequence $H_{j}^{m}, j=1,2 \ldots$ that is dense in the space of all $m$-spheres in $H^{n}$. Then every convex subset $S$ that is approximately $\ell$-dimensional for $\ell>k$ will be cut infinitely often by $h$ 's almost normal to it, thus making it $\ell$-dimensional width going to zero for $i \rightarrow \infty$; therefore, some sequence of finite partitions $\pi_{i}$ converges, for $i \rightarrow \infty$ to the desired $\pi$. Q.E.D. 


\subsection{Construction of $c_{0}$ and the proof of the waist theorem in the Gaussian case}

The above corollary does not directly imply 4.4.A, since the map $c_{\bullet}$, unlike $c_{\bullet}^{k}$ in 4.4, is supposed to be continuously defined for all convex subsets $S$ not only of those with codim $S=k$. In some cases, one can construct a map $c_{\bullet}$ as in 5.7, that is arbitrarily close to $c_{\bullet}^{k}$ on the space of $k$-dimensional sets $S$ and then 5.7 does imply 4.4.A.

The easiest case is that of the Gaussian, or general 1-log-concave measure $\mu$ on $\mathbb{R}^{n}$ where one has the following well known

5.8.A Concavity property. Let $\mu$ be a 1-log-concave measure on a convex subset in $\mathbb{R}^{n}$ and let $U_{1}$ and $U_{2}$ be two convex domains. Consider the translates $U_{1}+x_{1}$ of $U_{1}$ for all $x_{1} \in \mathbb{R}^{n}$ and the intersections $U_{x_{1}}=\left(U_{1}+\right.$ $\left.x_{1}\right) \cap U_{2} \subset \mathbb{R}^{n}$. Then the function $\mu\left(U_{x_{1}}\right)$ is 1-log-concave $x_{1} \in \mathbb{R}^{n}$ in-so-far as it is $>0$.

Proof. The "middle intersection" $U_{y}$ for $y=\left(x_{1}+x_{2}\right) / 2$ contains the Minkowski half-sum $\frac{1}{2}\left(U_{x_{1}}+U_{x_{2}}\right)$ and the Brunn-Minkowski for log-concave measures applies.

Now we may use $c_{\bullet}=c_{\delta}$ by approximately extending $c_{\bullet}^{k}$ from $k$-dimensional subsets $S$ to $n$-dimensional ones using the partition of unity, for example, where such a $c_{\delta}$ is constructed in the $\delta$-neighbourhood of each $S$ of dimension $k$ and these extensions are brought together with a partition of unity (in the space of convex subsets in $\mathbb{R}^{n}$ ). The value of the resulting $c_{\delta}$ on the original $S$ equals a convex combinations of values of $c^{k}$ 's on the $k$-dimensional subsets $S^{\prime}$ close to $S$. Therefore, by the above concavity, the measures of the intersections of $S$ with the $\varepsilon$-ball around $s_{\bullet}=c_{\delta}(S)$ are almost as large as the original ones for $s=c_{\bullet}^{k}(S)$

$$
\mu\left(S \cap B\left(s_{\delta}, \varepsilon\right)\right) \geq \mu(S \cap B(s, \varepsilon))-\delta^{\prime},
$$

where $\delta^{\prime} \rightarrow 0$ for $\delta \rightarrow 0$. Thus 4.4.A is reduced to 5.7 and the proof of the log-concave (in particular, Gaussian) theorem follows.

\subsection{The spherical case}

The volume of the intersection of the $\varepsilon$-ball $B(x, \varepsilon) \subset S^{n}$ with a convex domain $U \subset S^{n}$ does not seem (?) a sufficiently concave function in $x \in S^{n}$ in order to apply the above argument. However, this volume

$$
v_{\varepsilon}(x)=\mu(U \cap B(x, \varepsilon))
$$


is, obviously, weakly concave in the following sense: the value of $v_{\varepsilon}$ at each point $y$ on the geodesic segment $\left[x_{1}, x_{2}\right] \subset S^{n}$ satisfies

$$
v_{\varepsilon}(y) \geq C_{n} \min \left(v_{\varepsilon}\left(x_{1}\right), v_{\varepsilon}\left(x_{2}\right)\right)
$$

for some $C_{n}>0$ (possibly $C_{n}=1$ ).

When we approximately extend $c_{\bullet}^{k}$ from $k$-dimensional to $n$-dimensional sets, we face a (convex) ambiguity, only at those $k$-dimensional $S$ that are close to $(k-1)$-dimensional ones. Then the volume $v_{\varepsilon}\left(c_{\bullet}^{k}(S)\right)$ becomes of order $\varepsilon^{k-1}$ rather than $\varepsilon^{k}$, and in the limit, we either have no ambiguity problem for almost all $S_{\pi}, \pi \in \Pi$, or we arrive at some level $Y_{z}=f^{-1}(z)$ with infinite Minkowski measure of codimension $k$. The latter can be ruled out by approximating $f$ by generic smooth maps $f^{\prime}: S^{n} \rightarrow \mathbb{R}^{k}$ and applying the above to these $f^{\prime}$. The spherical waist theorem for $f$ itself follows by letting $f^{\prime} \rightarrow f$.

\section{6 (Co)homological partition theorems}

The remaining problem in the proof of the general case of 4.4.A is due to the ambiguity in approximation of a convex domain $U \subset S^{n}$ by a $k$ dimensional convex subset $S$ for some $k<n$. This ambiguity is of the same nature as the selection of the first eigenvector of a symmetric matrix at a point with multiple eigenvalues, since the above approximation reduces to a choice of $k$ principle eigenvectors for the measure (distribution) $\mu=\mu_{U}$ associated to $U$ that is assumed to have a positive continuous density in $U$. In general, given a probability measure $\mu$ on $\mathbb{R}^{n}$ with $n$-dimensional support (or rather with the convex hull of the support of dimension $n$ ) one uses the canonical scalar product on affine functions $\mathbb{R}^{n} \rightarrow \mathbb{R}$, that defines an elliproid $E=E(U)$, (approximating $\mu$ ) centered at the center of mass $c(\mu) \in \mathbb{R}^{n}$ of $\mu$ that is the unit ball of the dual scalar product (Hilbertian norms) on $\mathbb{R}^{n}$. Let $\ell_{1}, \ell_{2}, \ldots, \ell_{n}$ be the principal axes of $E$ written in the length decreasing order and let $L_{k} \subset \mathbb{R}^{n}$ be an affine space spanned by $\ell_{1}, \ldots, \ell_{k}$ (that passes through $c(\mu)$ ). The relevant $k$-dimensional measure $\mu_{k}$ (traditionally) used as a $k$-dimensional approximation to $\mu$ is (defined to be) the normal projection (push-forward) of $\mu$ to $L_{k}$.

The choice of $L_{k}$, and thus of $\mu_{k}$ become ambiguous if the $k$-th eigenvalue of (the quadratic form associated to) $E$ is non-simple: multiplicity of order $\delta$ brings forth $(\delta-1)$-pencil (projective space) of $L_{k}$ 's. However, this is not so bad as it looks since ellipsoids (i.e. symmetric matrices) with multiple eigenvalues have codimension two (not one!) in the space of all ellipsoids 
$E$. Thus, for a generic family of measures $\mu_{v}$, parametrized by, say, a closed $d$-manifold $V \ni v$, the totality of $L_{k}=L_{k}\left(\mu_{v}\right)$ make a $d$-cycle: the space $\mathcal{L}$ of pairs $\left(v, L\left(\mu_{v}\right)\right) \subset V \times \mathrm{Gr}_{k}\left(\mathbb{R}^{n}\right)$, (where $\mathrm{Gr}_{k}$ stands for the Grassmann manifolds of $k$ planes in $\mathbb{R}^{n}$ ) is a $d$-cycle, where the "cycle" property follows from the local real algebraic origin of $\mathcal{L}$ and where the projection of $\mathcal{L}$ to $V$ is non-homologous to zero mod 2 in $V$. Consequently, the corresponding cohomology homomorphism $H^{*}\left(V ; \mathbb{Z}_{2}\right) \rightarrow H^{*}\left(\mathcal{L} ; \mathbb{Z}_{2}\right)$ does not vanish. This remains true for all subsets $U \subset V$ : the homomorphism from $H^{*}\left(U ; \mathbb{Z}_{2}\right)$ to the cohomology of the pull-back of $U$ in $\mathcal{L}$ (for the projection $\mathcal{L} \hookrightarrow$ $\left.V \times \mathrm{Gr}_{k}\left(\mathbb{R}^{n}\right) \rightarrow V\right)$ is injective. This goes along with the non-vanishing lemma and a simple generalization of the proof of 5.7 yields 4.4.A. In fact, the (co)homological nature of this argument leads to the following generalization of 4.4 .

\subsection{Borsuk-Ulam families of cycles}

A BU-family of (codimensoion $k$ "cycles") subsets in $S^{n}$ is given by the following data

(i) A topological space $\widetilde{X}$ with a continuous map $p: \widetilde{X} \rightarrow S^{n}$

(ii) A continuous map $f$ from $\widetilde{X}$ to a space $Z$.

The resulting "cycles" are the images $Y_{z} \subset S^{n}$ of the pull-backs $\tilde{Y}_{z}=$ $f^{-1}(z) \subset \widetilde{X}$ under $p: \widetilde{X} \rightarrow S^{n}$, i.e. $Y_{z}=p\left(f^{-1}(z)\right) \subset S^{n}, z \in Z$.

We assume that the cohomology homomorphism $\mathbb{Z}_{2}=H^{n}\left(S^{n}, \mathbb{Z}_{2}\right) \rightarrow$ $H^{n}\left(\widetilde{X} ; \mathbb{Z}_{2}\right)$ is injective and that the parameter space $Z$ is a $k$-dimensional $\mathbb{Z}_{2}$-homology manifold (e.g. a topological $k$-manifolds). This means that $Z$ satisfies the local Poincaré duality over $\mathbb{Z}_{2}$. For example, if $Z$ is a polyhedral space, then the link of each $m$-simplex has the same $\mathbb{Z}_{2}$-homology as $(n-m-1)$-sphere. Equivalently, the "normal bundle" of the diagonal $\Delta_{Z} \subset Z \times Z$ has the structure of a $\mathbb{Z}_{2^{-}}$"vector" bundle. In particular, it possesses a coherent system of local Thom isomorphisms and well defined Stiefel-Whitney classes.

\subsection{UB-partition theorem}

If $c_{0}^{k}$ and $C_{\pi}$ are as in 4.4 and $k<n$ then the conclusion of 4.4.A holds true for every UB-family: there exists a convex partion $\Pi$ of $S^{n}$ into (at most) $k$-dimensional subsets $S_{\pi}$ such that some $Y_{z}, z \in Z$ meets $C_{\pi} \subset S_{\pi}$ for almost all $\pi \in \Pi$. 
Remark. The case $k=n$, corresponding to Borshuk-Ulam theorem, needs extra assumptions on $Z$ and/or on $f$. For example, it suffices to assume that $Z$ splits into $Z_{0} \times \mathbb{R}$.

Sketch of the proof. To clarify the geometry, let $Z$ be a $k$-manifold. Take an $n$-manifold $\widetilde{X}$ and a generic smooth map $f: \widetilde{X} \rightarrow Z$. Then the pull-back of the diagonal under the square $f \times f: \widetilde{X} \times \widetilde{X} \rightarrow Z \times Z$, say $\widetilde{\Delta}=(f \times f)^{-1}\left(\Delta_{Z}\right) \subset \widetilde{X} \times \widetilde{X}$ makes a relative $(2 n-k)$-cycle in $\left(\tilde{X} \times \tilde{X}, \Delta_{\tilde{X}}\right)$, whose $\mathbb{Z}_{2}$-equivariant homology class is invariant under homotopies of maps $\widetilde{X} \rightarrow Z$ (and under homotopies of $\mathbb{Z}_{2}$-equivariant maps $\widetilde{X} \times \widetilde{X} \rightarrow Z \times Z$ in general). Next, consider a smooth map $p: \widetilde{X} \rightarrow S^{n}$, take a round (not necessarily equatorial) sphere $S^{k} \subset S^{n}$ and look at the cycle $\widetilde{S}^{k} \subset \widetilde{X} \times \widetilde{X} \backslash \Delta_{\widetilde{X}}$ that is the pull-back under $p \times p$ of the set of the pairs of opposite points in $\widetilde{S}^{k}$.

6.2.A. If $p$ has non-zero $\mathbb{Z}_{2}$-degree and $k<n$, then the $\mathbb{Z}_{2}$-intersection between $\widetilde{\Delta} / \mathbb{Z}_{2}$ and $\widetilde{S}^{k} / \mathbb{Z}_{2}$ in $\widetilde{X} \times \widetilde{X} / \mathbb{Z}_{2}$ is non-zero $\bmod 2$.

Proof. If $S^{k}$ is a small sphere at some point $s \in S^{n}$, this amounts to the (homological content of the) Borsuk-Ulam theorem. Since $k<n$, all $k$-spaces are homotopic in $S^{n}$ and the proof follows by the invariance of $\mathbb{Z}_{2}$-intersection numbers under homotopies.

The non-vanishing of this intersection (expressed in the cohomological language) is sufficient for carrying over the argument in 5.7 and (with some homological tinkering) the proof of 6.2 follows.

\subsection{Examples and applications}

Let $\widetilde{X}=\widetilde{Y} \times Z$ where $p: \widetilde{X} \rightarrow Z$ is the projection.

If $\operatorname{dim} Z=k$, the conclusion of 6.2 holds true without assuming that $Z$ is a $\mathbb{Z}_{2}$-manifold.

In fact, if $Z$ is a polyhedron, then every homology class in it is realizable by a mapped manifold $Z^{\prime} \rightarrow Z$. Then 6.2 applies to the product $\widetilde{X}^{\prime}=$ $\widetilde{Y} \times Z^{\prime} \rightarrow Z^{\prime}$. Since general, say compact, spaces $Z$ can be approximated by polyhedra, we obtain the same result for all $Z$.

One easily extends the above to the case where $p: \widetilde{X} \rightarrow Z$ is an arbitrary (not necessarily trivial) fibration or even a topological submersion. In fact, one needs the fibration (submersion) condition only over those points $z \in Z$, where the $\mathbb{Z}_{2}$-manifold condition fails to be satisfied. 
Remark. In most examples $Z$ comes as a manifold; we brought in $\mathbb{Z}_{2}$-manifolds in order to emphasize the (local) (co)homological aspect of the Ulam-Borsuk theorem. If $Z$ a general, say compact $k$-dimensional space one can obtain lower bounds on waists (and/or concentration) by just topologically embedding $Z$ to a $2 k$-manifold $Z_{+} \supset Z$, but these do not seem sharp for $S^{n}$ and $\mathrm{Ga}^{n}$. On the other hand, if $k=1$, then, e.g. for $Z$ being a tree, one arrives at the isoperimetric problem of estimating the volume of the $\varepsilon$-neighbourhood of a subset $Y$ in $X$ (e.g. $X=S^{n}$ ) dividing $X$ into three parts of given volumes. This can be reduced in some cases (e.g. for 1-logconcave measures in $\mathbb{R}^{n}$ ) to a 2 -dimensional problem (by using the partition argument from [Gro-Mil] with Borshuk-Ulam applied to maps $S^{2} \rightarrow \mathbb{R}^{2}$, rather than to $S^{1} \rightarrow \mathbb{R}^{1}$ where an explicit solution seems easy.

Question. Does $(\star)_{S^{n}}$ hold true for all $k$-dimensional spaces $Z$ (not just for $\mathbb{Z}_{2}$-manifolds such as $\mathbb{R}^{k}$ and/or for $n$-dimensional manifolds $\widetilde{Z}$ mapped to $S^{n}$ with even degree $\neq 0$ ?

6.3.A Isometric UB-families. Take a $((n-k)$-dimensional) space $\tilde{Y}$, a continuous map $p_{0}: \widetilde{Y} \rightarrow S^{n}$ and all "isometric translates" of this $\widetilde{Y} \rightarrow S^{n}$; thus $\widetilde{X}=\widetilde{Y} \times S O(n+1)$ and $p: \widetilde{X} \rightarrow S^{n}$ is defined by $p(\widetilde{y}, z)=z p(\widetilde{y})$ for all $\widetilde{y} \in Y$ and $z \in S O(n+1)$.

If $\tilde{Y}=S^{n-k}$ and the map $p_{0}: \tilde{Y}=S^{n-k} \rightarrow S^{n}$ is equivariant for the standard involution on the spheres, then the family $Y_{z}=z p_{0}\left(S^{n-k}\right) \subset S^{n}$ admits a partition as in 6.2 provided $n$ is odd and $k=2^{m}-1$ for some $m=1,2 \ldots$

Proof. We need a $k$-imensional cycle $Z^{\prime} \subset S O(n)$, such that the map $p$ restricted to $\widetilde{X}^{\prime}=\widetilde{Y} \times Z^{\prime}$ is non-homologous to zero $\bmod 2$. We take this $Z^{\prime}$ inside $S O(k+1)$ by rotating $S^{k} \subset S^{n}$ where we pass to the projective spaces $P^{k}=S^{k} / \mathbb{Z}_{2} \subset P^{n}=S^{n} / \mathbb{Z}_{2}$ and observe that for $n$ odd, the existence of $Z^{\prime}$ reduces to surjectivity of the homology homomorphism for the full tangent frame bundle $F_{k}$ over $P^{k}$. Since the Stiefel-Whitney classes of $P^{k}$ for $k=2^{m}-1$ vanish, the frame fibration $F_{k} \rightarrow P^{k}$ (with fibers $S O(k)$ ) is surjective on the $\mathbb{Z}_{2}$-homology. Q.E.D.

6.3.B Corollary. The volumes of the $\varepsilon$-neighbourhoods of $Y_{0}=p_{0}\left(\widetilde{Y}=S^{k}\right)$ in the standard round sphere $S^{n}$ are bounded from below by those of the equatorial $S^{n-k} \subset S^{n}$, provided $n$ is odd and $k=2^{m}-1$. 
Remarks and questions. (a) Doesn't it look bizarre? Conjecturally, the conclusion of 6.3.B holds true for all $n$ and $k$ : an $(n-k)$-cycle $Y$ in the projective space $P^{n}$ non-homologous to zero must have $\operatorname{Vol}(Y+\varepsilon) \geq$ $\operatorname{Vol}\left(P^{n-k}+\varepsilon\right)$ for all $\varepsilon>0$, not only for $P^{n}=\mathbb{R} P^{n}$ but for $\mathbb{C} P^{n}$ and $\mathbb{H} P^{n}$ as well. Moreover, such lower bound (in a non-sharp form) might be true for non-Riemannian metrics in $P^{n}$ 's associated to some (all?) Banach norms in $\mathbb{R}^{n+1}\left(\mathbb{R}^{n+2}\right.$ for $\mathbb{C}$ and $\mathbb{R}^{n+4}$ for $\left.\mathbb{H}\right)$, where the main issue is the asymptotics of (the constant in) such a bound for $n \rightarrow \infty$. (A lower bound for $\operatorname{Haumes}(Y)$ is covered by isosystolic inequalities in [Gro $]_{F R M}$ where the constants are very poor but dimension free.) And it would not hurt to have such inequalities for other symmetric spaces, Grassmann manifolds $\mathrm{Gr}_{m} \mathbb{R}^{n}$ for instance (that may carry non-homogeneous metrics associated to non-Euclidean norms on $\left.\mathbb{R}^{n}\right)$.

(b) A geometric characteristics close to the waist arises when one takes the square of a map $f: X \rightarrow Z$ and looks at the measures of the $\varepsilon$ neighbourhoods of te pull-backs of the diagonal, $\mu \times \mu\left((f \times f)^{-1}\left(\Delta_{Z}\right)\right)$, but it is unclear what are possible improvements over the waist inequalities (say for $X=S^{n}, Z=\mathbb{R}^{k}$ ). Next, one seeks configurations of $i$ points $x_{i} \in X$ mutually $\varepsilon$-apart, such that $f$ is constant on $\left\{x_{i}\right\} \subset X$. This suggests new metric invariants reflecting the "homological complexity" of the space of these $\varepsilon$-separated configurations in the space of all configuration of mutually distinct points, but it remains unclear what happens for specific $X$, not even for $X=S^{n}$.

6.3.C. Let $\widetilde{X}$ be an $n$-dimensional Riemannian manifold and suppose that the map $p: \widetilde{X} \rightarrow S^{n}$ (of non-zero $\mathbb{Z}_{2}$-degree) is $(n-k$ )-volume contracting, e.g. $p$ is $C^{1}$-smooth and the sup-norm of the differential of $p$ on the $(n-k)$ exterior power of the tangent bundle of $\widetilde{X}$, denoted $\Lambda^{k} d p: \Lambda^{k} T(\widetilde{X}) \rightarrow$ $\Lambda^{k}\left(S^{n}\right)$, is bounded by one: $\left\|\Lambda^{k} d p(\widetilde{x})\right\| \leq 1, \widetilde{x} \in \widetilde{X}$. Then some "fiber" $\widetilde{Y}_{z}=$ $f^{-1}(z) \subset \tilde{X}, z \in Z$, has its $k$-codimensional Minkowski volume bounded by that of the equatorial $S^{n-k} \subset S^{n}$, (where $Z$ is a $k$-dimensional $\mathbb{Z}_{2}$-homology manifold).

Proof. This follows from the lower bound on $\operatorname{Miwst}_{-k}\left(S^{n},\left\{Y_{z}=p\left(\tilde{Y}_{z}\right)\right)\right.$, where this bound is derived from the BU-partition theorem in the same way as $(\star)_{S^{n}}$.

Remark. If the "fibers" $\tilde{Y}_{z}, z \in Z$ are rectifiable then "Minskowski volume" can be substituted by the " $(n-k)$-dimensional Hausdorff measure" but this is unclear for (the "fibers" of) general continuous maps $f: \widetilde{X} \rightarrow Z$. 
6.3.D Filling and related problems. Let $\tilde{Y} \subset \mathbb{R}^{n+1}$ be an $(n-k)$ dimensional $\mathbb{Z}_{2}$-cycle, i.e. a $k$-submanifold with Fill $\operatorname{Rad}\left(\widetilde{Y} \subset \mathbb{R}^{n+1}\right) \geq 1$, i.e. such that $\tilde{Y}$ is not homologous to zero in its 1-neighbourhood $\tilde{Y}+1 \subset \mathbb{R}^{n+1}$. Then by taking $Z=\mathbb{R}^{n+1} \backslash(\widetilde{Y}+1)$ and radially projecting $\tilde{Y}$ to the unit spheres $S^{n}(z, 1)=S^{n}$ we conclude with 6.2 (compare $\S 8$ in [Gro] FRM) that $\widetilde{Y}$ admits a 1-Lipschitz map to $S^{n}$, say $p: \widetilde{Y} \rightarrow S^{n}$, such that the $\varepsilon$ neighbourhood of $p(\widetilde{Y}) \subset S^{n}$ have their spherical volumes $\geq \operatorname{Vol}\left(S^{n-k}\right)$ for all $\varepsilon>0$.

This, for $\varepsilon \rightarrow 0$, provides an elementary (with no use of calculus of variations) solution to the Gehring linking problem (see [Gro] $]_{\mathrm{FRM}}$ and references therein).

Remarks and questions. An anybody's guess is that the inequality Fill $\operatorname{Rad}\left(\tilde{Y} \subset \mathbb{R}^{n+1}\right) \geq 1$ implies that

$$
\operatorname{Vol}_{n+1}\left(\tilde{Y}+\varepsilon \subset \mathbb{R}^{n+1}\right) \geq \operatorname{Vol}_{n+1}\left(S^{n-k}+\varepsilon, \mathbb{R}^{n+1}\right)
$$

for the unit round $(n-k)$-sphere $S^{n-k} \subset \mathbb{R}^{n+1}$. (In fact one may expect such a bound on $\operatorname{Vol}_{n+1}(\widetilde{Y}+\varepsilon)$ under the assumption Fill Vol $\left(\widetilde{Y} \subset \mathbb{R}^{n+1}\right) \geq$ $\operatorname{Vol}_{n-k+1}\left(B^{n-k+1}(1)\right)$ for the unit $(n-k+1)$-ball in $\mathbb{R}^{n-k+1} \subset \mathbb{R}^{n+1}$, where the limit case for $\varepsilon \rightarrow 0$ is covered by Almgren's filling inequality.) The above does not yield such a bound since $\operatorname{Vol}(Y+\varepsilon)$ is not (always) decreasing under 1-Lipschitz maps. This suggests the following

Definition. (a) Given a metric space $Y$ and an $m m$ space $X$ let $\mu_{X}((Y \rightarrow X)+\varepsilon)$ stands for the supremum of the measures of the $\varepsilon$-neighbourhoods of the images of $Y$ under all 1-Lipschitz maps $Y \rightarrow X$.

The immediate question is that of identifying extremal subsets $Y$ in standard $X$ 's (such as $\mathbb{R}^{n}$ and $S^{n}$ ) i.e. those $Y \subset X$, where $\mu_{X}(Y+\varepsilon)=$ $\mu_{X}((Y \rightarrow X)+\varepsilon)$. This is related to a problem by Hadviger solved for $n=2$ in [Bez-Con].

(b) Let $Y_{1}$ and $Y_{2}$ be two disjoint cycles in $S^{n+1}$ of dimension $k$ and $(n-k)$ with non-zero linking number and with $\operatorname{dist}\left(Y_{1}, Y_{2}\right)=d$. What is the (sharp) lower bound on $\operatorname{Vol}\left(Y_{1}+\varepsilon\right)+\operatorname{Vol}\left(Y_{2}+\varepsilon\right)$ in terms of $d, \varepsilon_{1}$ and $\varepsilon_{2}$ ?

(c) The above filling bound for $\widetilde{Y} \subset \mathbb{R}^{n+1}$ extends to "sufficiently convex" non-Hilbertian Banach spaces $X \supset \widetilde{Y}$ (compare [Gro-Mil]) where it is much sharper, than the general filling theorem from [Gro $]_{\mathrm{FRM}}$ for $k$ small compared to $n$. 
Are there filling inequalities mediating between these two? What are the waists of the unit spheres $S(B)$ in the standard (finite dimensional) Banach spaces $B$ ? What are the $\varepsilon$-systoles of the corresponding projective spaces $P(B)=S(B) /\{-1,+1\}$ and other standard spaces (such as Grassmann manifolds) associated to $B$ ? (An $\varepsilon$-systole of an $m m$ space $X$ is defined as the ordinary one in [Gro $]_{\mathrm{SI}}$, where the $(n-k)$-Hausdorff measure of a cycle $Y \subset X$ is replaced by the volume of $Y+\varepsilon \subset X$.)

\section{$7 \quad$ Parametric partitions and $\mathbb{Z}_{2}$-waists}

Consider an $n$-sphere bundle $\alpha: X \rightarrow B$ and let $\mathcal{C}^{k}(X)$ denote the space of (exactly) $k$-dimensional convex subsets $S$ in the (spherical) fibers $S^{n}=$ $\alpha^{-1}(b) \subset X$ for all $b \in B$. Let $c^{k}: \mathcal{C}^{k}(X) \rightarrow X$ be a continuous ("center") map sending each $S \subset X$ to a point in $S$. Next consider a topological space $\widetilde{X}$ along with a map $p: \widetilde{X} \rightarrow X$ that is $\mathbb{Z}_{2}$-onto in the following sense: for every pair of compact subsets $X_{1} \subset X_{2} \subset X$ the $\mathbb{Z}_{2}$-cohomology homomorphism

$$
H^{*}\left(X_{2}, X_{1} ; \mathbb{Z}_{2}\right) \rightarrow H^{*}\left(p^{-1}\left(X_{2}\right), p^{-1}\left(X_{1}\right) ; \mathbb{Z}_{2}\right)
$$

is injective (and the corresponding homology homomorphism is surjective, provided the spaces in question are not overpathological). For example, if $X$ is a closed $\mathbb{Z}_{2}$-homology $N$-manifold, then $p$ is $\mathbb{Z}_{2}$-onto iff the homomorphism $H^{N}\left(X ; \mathbb{Z}_{2}\right) \rightarrow H^{N}\left(\widetilde{X}, \mathbb{Z}_{2}\right)$ is injective. Another instance of this, recurrent in the present paper, is the full (or partial) non-oriented flag bundle of a real vector bundle, where the $\mathbb{Z}_{2}$-onto property amounts to the splitting principle for $O(n)$-bundles.

Take, furthermore, a fibration $\beta: Z \rightarrow B$, where the fibers are $k$ dimensional $\mathbb{Z}_{2}$-homology manifolds. (In truth, $\beta$ does not need to be a fibration: topological submersions will do. Moreover, one can allow maps $\varphi$ with a suitable Poincaré duality satisfied by their Leray cohomology sheaves) and let $f: \widetilde{X} \rightarrow Z$ be a fiberwise (i.e. $\beta f=\alpha f$ ) continuous map.

Now, let us restrict attention to what happens over a given point $b \in B$, where we have the fibers $X_{b}=S^{n}, \widetilde{X}_{b}$ and $Z_{b}$ along with the maps $p_{b}$ : $\widetilde{X}_{b} \rightarrow X_{b}, f_{b}: \widetilde{X}_{b} \rightarrow Z_{b}$, and $c_{b}^{k}: \mathcal{C}_{b}^{k}=C^{k}\left(X_{b}\right) \rightarrow X_{b}$. One checks easily that these satisfy the assumption of 6.2 and therefore, there exists $z_{b} \in Z_{b}$, such that the level $Y_{z_{b}}=p_{b} f^{-1}\left(z_{b}\right) \subset X_{b}$ meets the (central) subsets $C_{\pi_{b}}$ of some convex partition $\Pi_{b}$ of $X_{b}=S^{n}$ with (at most) $k$-dimensional "slices" $S_{\pi} \subset X_{b} \pi \in \Pi_{b}$. 
Denote by $Z_{b}^{\prime} \subset Z_{b}$ the set of all these $z_{b} \in Z_{b}$, let $Z^{\prime} \subset Z$ be the union of $Z_{b}^{\prime}$ over all $b \in B$ and $\beta^{\prime}: Z^{\prime} \rightarrow B$ be the restriction of $\beta: Z \rightarrow B$ to $Z^{\prime}$.

\subsection{Parametric BU-partition theorem}

The map $\beta^{\prime}$ is $\mathbb{Z}_{2}$-onto.

Proof. If $B$ consists of a single point $b$ this is equivalent to 6.2 claiming that $Z^{\prime}=Z_{b}^{\prime}$ is non-empty in this case. The $\mathbb{Z}_{2}$-onto property says, in effect, that $Z_{b}^{\prime}$ is "(co)homologically continuous" in $b \in B$ for all $B$. In fact, the formal structure of the proof of 6.2 automatically ensures such "continuity" (or functoriality) and the proof of 7.1 follows.

\subsection{Remark: $\mathbb{Z}_{2}$-morphisms}

One can save notations by introducing the (well known, I guess) notion of a $\mathbb{Z}_{2^{-}}$(faithful)-morphism $\widetilde{f}: X \rightarrow Z$ that is (a multivalued map) given by a pair $p: \widetilde{X} \rightarrow X$ and $f: \widetilde{X} \rightarrow Z$ with $p$ being $\mathbb{Z}_{2}$-onto. (One can "reduce" such a morphism to a correspondence $\bar{f}: X \rightarrow Z$ by taking the image $\bar{X} \subset X \times Z$ of the map $p \times f: \widetilde{X} \rightarrow X \times Z$.)

Example. If $X$ is a smooth real algebraic variety, then every rational map $X \rightarrow Z$ is a $\mathbb{Z}_{2}$-morphisms, where $Z$ is another (possibly singular) real algebraic variety and where "smooth" can be relaxed to " $\mathbb{Z}_{2}$-homology manifold". The $\mathbb{Z}_{2}$-morphisms make a decent category, where one avoids explicitly mentionning $\widetilde{X}$ 's. (Also the center maps $c^{k}: C \rightarrow S^{n}$ can be replaced by a $\mathbb{Z}_{2}$-morphisms without changing the conclusions of the partition theorems.) For instance, the Borsuk-Ulam theorem obviously applies to $\mathbb{Z}_{2}$-morphisms $\tilde{f}: S^{n} \rightarrow \mathbb{R}^{n}$ and claims that $\tilde{f}(s) \cap \widetilde{f}(-s) \neq \emptyset$ for some $s \in S^{n}$ (for $\left.\tilde{f}(s)=f\left(p^{-1}(s)\right) \subset \mathbb{R}^{n}\right)$. More generally, let $\alpha: X \rightarrow B$ be a (topological) $n$-sphere bundle with a fixed point free fiberwise continuous involution, denoted $x \mapsto-x, x \in X$, let $\beta: Z \rightarrow B$ be a topological submersion (e.g. a locally trivial fibration), where the fibers are $\mathbb{Z}_{2}$-homology $k$-manifolds, and let $\tilde{f}: X \rightarrow Z$ be a $\mathbb{Z}_{2}$-morphisms over $B$, i.e. the implied map $f: \widetilde{X} \rightarrow Z$ sends each "fiber" $\widetilde{X}_{b}=(\alpha \circ p)^{-1}(b)$ to $Z_{b}, b \in B$. Denote by $\mathrm{BU}=\mathrm{BU}(\widetilde{f}) \subset \widetilde{X} \times \widetilde{X}$ the subset of the pairs $\left(\widetilde{x}_{1}, \widetilde{x}_{2}\right)$ satisfying the equations

$$
p\left(\widetilde{x}_{1}\right)=-p\left(\widetilde{x}_{2}\right) \quad \text { and } \quad f\left(\widetilde{x}_{1}\right)=f\left(\widetilde{x}_{2}\right) .
$$


Parametric BU-theorem. If $k<n$ (or $k=n$ and the fibers $Z_{b}$ are contractible) then the (tautological) projection $\mathrm{BU} \rightarrow B$ is $\mathbb{Z}_{2}$-onto. Consequently, the $\tilde{f} \times \widetilde{f}$-image $\widetilde{f} \times \widetilde{f}(\mathrm{BU}) \subset Z$ projects $\mathbb{Z}_{2}$-onto $Z$.

This is obvious (in the $21^{\text {th }}$ century).

Corollary. Let $f_{b}: S^{n} \rightarrow Z, b \in B$, be a continuous family of maps. Then the assignement to each $b \in B$ of (the subset of) those $z \in Z$ for which $\exists s \in S$, where $f_{b}(s)=f_{b}(-s)=z$, is a $\mathbb{Z}_{2}$-morphism.

Intuitively, one can pretend that the equation $f_{b}(s)=f_{b}(-s)$ has a unique, up-to $\pm \operatorname{sign}$, solution $s=s(b)$ continuously depending on $b \in B$ and $z(b)=f(s(b))$ also unique and continuous in $b$.

\subsection{Parametric waists}

Consider a family $X=\left\{X_{b}\right\}, b \in B$ of $m m$ spaces, that is a (continuous) map $\alpha: X \rightarrow B$ with metrics dist ${ }_{b}$ and measures $\mu_{b}$ in the "fibers" $X_{b}=\alpha^{-1}(b)$, $b \in B$, and a (double) family of " $k$-cycles" $Y_{z_{b}} \subset X_{b}$ defined with $\beta: Z \rightarrow B$ by an $\tilde{f}: X \rightarrow Z$, where $\widetilde{f}$ refers to some space $\widetilde{X}$ mapped to $X$ and to $Z$, that are $p: \widetilde{X} \rightarrow X$ and $f: \widetilde{X} \rightarrow Z$; here $\tilde{f}$ is a fiber preserving map (over $Z$ ) and $Y_{z_{b}}=p\left(\widetilde{f}^{-1}\left(z_{b}\right)\right) \subset X_{b} \subset X$ for $z_{b} \in Z_{b}=\beta^{-1}(b) \subset Z$. Given a function $w(b, \varepsilon), b \in B, \varepsilon \geq 0$, let $Z^{\prime}=Z^{\prime}(w) \subset Z$ consist of those $z_{b} \in Z_{b} \subset Z, b \in B$, where

$$
\mu_{b}\left(Y_{z_{b}}+\varepsilon\right) \geq w(b, \varepsilon)
$$

for all $\varepsilon \geq 0$.

Say that the $\mathbb{Z}_{2}$-waist of the family $\left\{Y_{z_{b}}\right\}$ is $\geq w=w(b, \varepsilon)$ it the restriction of $\beta: Z \rightarrow B$ to $Z^{\prime} \subset Z$ is $\mathbb{Z}_{2}$-onto. Then we write

$$
\mathbb{Z}_{2^{-}} \operatorname{wst}(X \underset{\beta}{\rightarrow} Z, \varepsilon) \geq w(b, \varepsilon)
$$

if for every $\mathbb{Z}_{2}$-morphism $\tilde{f}: X \rightarrow Z$ over $B$, (defined via an $\tilde{X}$ and $f: X \rightarrow$ $Z$ as above with $p: \widetilde{X} \rightarrow X$ being $\mathbb{Z}_{2}$-onto)

$$
\mathbb{Z}_{2^{-}} \text {wst }\left\{X_{z_{b}}\right\} \geq w
$$

If we deal with individual $X_{0}$ and $Z_{0}$, we consider arbitrary fibrations $\alpha: X \rightarrow B$ and $\beta: Z \rightarrow B$ with the fibers $X_{b}$ and $Z_{b}$ isomorphic to $X_{0}$ and $Z_{0}$ respectively where "isomorphism" refers to "homeomorphism" for $Z_{b}$ and to the measure preserving isometries for $X_{b}$; moreover we insist in 
the latter case on $\alpha$ being a MIs $\left(X_{0}\right)$-fibration for the group $\operatorname{MIs}\left(X_{0}\right)(m m$ preserving isometries of $X_{0}$ ). (If $X_{0}$ is an $m m$-space, where the metric is allowed to become infinite, "homeomorphisms" refer to a topological structure additionally given to $X_{0}$, as for infinite Cartesian powers of compact $m m$-spaces, for example.) We say that the parametric $\mathbb{Z}_{2}$-waist of $X_{0} \rightarrow Z_{0}$ is $\geq w(\varepsilon)$,

$$
\mathbb{Z}_{2^{-}} \operatorname{pawst}\left(X_{0} \rightarrow Z_{0}, \varepsilon\right) \geq w(\varepsilon)
$$

if

$$
\mathbb{Z}_{2^{-}} \operatorname{wst}(X \underset{\beta}{\longrightarrow} Z, \varepsilon) \geq w(\varepsilon)
$$

for all fibrations $X$ and $Z$ over all $B$ with the fibers $X_{0}$ and $Z_{0}$ respectively. Similarly, one defines the Gibbsian versions $\mathbb{Z}_{2}$-Giwst and $\mathbb{Z}_{2}$-paGiwst.

\section{$7.4 \mathbb{Z}_{2}$-waists of product and fibrations}

Let a $p m$ space $X^{\prime}$ be fibered over $X$ with fibers $X_{x}^{\prime}, x \in X$, where $X$ is given the pushforward $\mu$ of the measure $\mu^{\prime}$ on $X$ and the fibers come with the canonical (probability) measures $\mu_{x}^{\prime}$. Consider the family of codimension $k$-cycles $Y_{z}^{\prime}=f^{-1}(z)$ for a continuous map $f: X \rightarrow Z$ and assume that

$$
\mathbb{Z}_{2^{-}} \operatorname{wst}\left(X^{\prime} \underset{X}{\longrightarrow} Z, \varepsilon\right) \geq w(\varepsilon)
$$

for some function $w^{\prime}=w^{\prime}(\varepsilon)$. This means, by the definition of the $\mathbb{Z}_{2}$-waist over $X$, that there is a " $\mathbb{Z}_{2}$-homological selection" of $z=z(x) \in Z$ for all $x \in$ $X$, where the corresponding $Y_{z(x)}=f_{x}^{-1}(z) \in X_{x}^{\prime}$ have $\mu_{x}^{\prime}\left(Y_{z(x)}+\varepsilon\right) \geq w^{\prime}(\varepsilon)$ (and where the ambiguity of this selection makes the correspondence $x \mapsto$ $z(x)$ a $\mathbb{Z}_{2}$-morphism rather than an ordinary map). Granted the inequality $\mathbb{Z}_{2^{-}}$wst $(X \rightarrow Z, \varepsilon) \geq w(\varepsilon)$, we apply it to the above $\mathbb{Z}_{2}$-morphism $X \rightarrow Z$ and obtain a subset $\underline{Y} \subset X$ with $\mu(\underline{Y}+\varepsilon) \geq w(\varepsilon)$, where the "fibers" $Y_{z(x)}^{\prime}=f^{-1}(z) \cap X_{x}^{\prime}$ have $\mu_{x}^{\prime}\left(Y_{z(x)}^{\prime}+\varepsilon\right) \geq w^{\prime}(\varepsilon)$ for all $x \in \underline{Y}$. Then, under suitable conditions on the metric in $X^{\prime}$ we obtain a lower bound on $\mu^{\prime}\left(f^{-1}(z)+\varepsilon\right)$ in $X^{\prime}$ and thus on wst $\left(X^{\prime} \rightarrow Z\right)$. Furthermore, if the waists of $X^{\prime}$ over $X$ and of $X$ are bounded from below in the $\mathbb{Z}_{2}$-parametric form, then such a bound for $X$ also applies to $\mathbb{Z}_{2}$ - pawst $(X, \varepsilon)$. Similarly, one treats the Gibbsian $\mathbb{Z}_{2}$-waists and immediately obtains the product inequalities $(x)_{\ell_{\infty}}$ and $(x)_{\mathrm{Gi}}$ in 3.3, as well as the same inequality for metric fibrations, e.g. (classical) Riemannian fibrations in the Gibbsian case. (We leave to the reader working out specific metric assumptions on $X^{\prime} \rightarrow X$ allowing lower product waist bound for $X^{\prime}$ in terms of $X$ and the fibers.) 


\subsection{Pawst inequalities}

It follows from the above discussion that all waist inequalities stated in 1 remain valid with $\mathbb{Z}_{2}$-pawst's instead of wst's, and, whenever the "central" map $c_{\bullet}^{k}$ appears, it may be assumed a $\mathbb{Z}_{2}$-morphism rather than an ordinary map. Moreover, one can generalize the waist inequality to sphere bundles over $B$, where the measure may vary with the fiber. Then the essence of the partition proof of the waist inequalities can be seen in the following

Subdivision lemma. Let $\mu$ be a measure on $S^{n}$ with positive continuous density and $H$ denote the space of oriented hyperplanes (equators) $h$ dividing $S^{n}$ into halves of equal $\mu$-measure, say $X_{h}^{+}$and $X_{h}^{-}$. The totalities of these make fibrations $X^{+}$and $X^{-}$over $H$ with half-sphere fibers. Then the $\mathbb{Z}_{2^{-}}$ waist of $\left(S^{n}, \mu\right)$ is bounded from below by the sum of the $\mathbb{Z}_{2}$-waists of $X^{+}$ and $X^{-}:$if $\mathbb{Z}_{2^{-}} \operatorname{wst}\left(X^{ \pm} \underset{H}{\longrightarrow} Z, \varepsilon\right) \leq w^{ \pm}(h, \varepsilon)$, then

$$
\mathbb{Z}_{2^{-}} \operatorname{wst}\left(\left(S^{n}, \mu\right) \rightarrow Z, \varepsilon\right) \geq \inf _{h \in H}\left(w^{+}(h, \varepsilon)+w^{-}(h, \varepsilon)\right) .
$$

This is an immediate corollary of the Borsuk-Ulam theorem that remains valid for sphere bundles instead of an individual $\left(S^{n}, \mu\right)$. It can be applied repeatedly eventually reducing the waist estimate for $\mu$ to that for families of convexly derived measures on convex subsets of codimension $\geq k$ in $S^{n}$.

\section{Waists of degree $d \geq 1$}

The property of a family of subsets $\{Y \subset X\}$ essential for lower waist bounds says, in effect, that there is a " $\mathbb{Z}_{2}$-essentially unique" $Y$ in the family containing every given point in $X$. More precisely, say that the family is $\mathbb{Z}_{2}$-faithful in codimension $k$, or it admits a $\mathbb{Z}_{2}$-faithful $k$-parametrization (by a $Z$ ) if there exists a $\mathbb{Z}_{2}$-morphism $f$ from $X$ to a $\mathbb{Z}_{2}$-homology manifold $Z$, such that each $Y_{z} \subset X$ associated to $f$ (that is $\left.p\left(\widetilde{f}^{-1}(z)\right)\right)$ is contained in some $Y$ in the family. This eventually applies to the parametric case and the result is denoted $\mathbb{Z}_{2^{-}}$pawst $(X, \varepsilon)$ : this is essentially $\inf _{Z} \mathbb{Z}_{2^{-}}$pawst $(X \rightarrow Z, \varepsilon)$ over all $\mathbb{Z}_{2}$-homology $k$-manifolds $Z$.

Given a family $\{Y\}$ in $X$ denote by $\left\{Y^{d} \subset X^{d}\right\}$ the family of the $d$-th Cartesian powers of $Y$ 's and say that the original family is $\mathbb{Z}_{2}$-faithful of degree $d$ in codimension $k$ if $\left\{Y^{d}\right\}$ is $\mathbb{Z}_{2}$-faithful in codimension $k d$. 


\subsection{Examples}

Let $X$ be a smooth real algebraic variety and consider a family of subvarieties $Y \subset X$ with $\operatorname{codim} Y=k$. If the number of $Y$ 's passing through a generic $d$-tuple of points in $X$ is finite and odd, then this family is $\mathbb{Z}_{2}$-faithful of degree $d$ in codimension $k$. (Here, a family, presupposes that each $Y$ is a limit of generic members of the family.)

An instance of this is the family of all $d$-tuples of points in $X$ (of dimension $k)$. Another relevant case is that of all $(n-k)$-planes in $\mathbb{R}^{n}$ or in $P^{n}$ that is (obviously) faithful of degree $(n-k)$ in codimension $k$.

\subsection{Induced families}

Given a family of $Y$ 's in $Z$ and a map $f: X \rightarrow Z$ one has the induced family $f^{-1}\{Y\}$ in $X$. If $\{Y\}$ is faithful then $\left\{f^{-1}(Y)\right\}$ is also faithful with the same codimension and degree; moreover this remains true for an arbitrary $\mathbb{Z}_{2}$-morphism $X \rightarrow Z$.

\subsection{Semiadditivity of wst $^{d}$}

Now we introduce with our standing convention the waist $\mathbb{Z}_{2}$ - pawst $_{-k}^{d}(X, \varepsilon)$, abbreviated to wst $_{-k}^{d}(X)$, with the above faithful families and observe that every pair of disjoint open subsets $X_{1}$ and $X_{2}$ in $X$ satisfies

$$
\mathrm{wst}_{-k}^{d}(X) \geq \mathrm{wst}_{-k}^{d_{1}}\left(X_{1}\right)+\mathrm{wst}_{-k}^{d_{2}}\left(X_{2}\right) \quad(+)_{d}
$$

for every $k$ and all $d_{1}, d_{2}$ with $d_{1}+d_{2}=d$.

Proof. This automatically follows from the $\mathbb{Z}_{2}$-parametric definition of wst $^{d}$.

The above applies $d$-times to a partition of $X$ into $d$ small pieces $X_{i}, i=$ $1, \ldots, d$ with diameters $D_{i} \lesssim((\operatorname{Vol} X) / d)^{\frac{1}{n}}$ for large $d$, thus bounds wst $^{d}(X)$ by $\sum_{i=1}^{d} \mathrm{wst}^{d}\left(X_{i}\right)$. The latter waist, for small "roundish" $X_{i}$ can be bounded from below, with a Gaussian domination for example, by $\approx D_{i}^{n-k} \varepsilon^{k}$ provided $\varepsilon \leq D_{i}$. In particular, the Minkowski waist of $X$ satisfies

$$
\liminf _{d \rightarrow \infty} d^{\frac{k-n}{n}} \operatorname{Miwst}_{-k}^{d}(X) \geq C_{k, n}^{-}(\operatorname{Vol} X)^{\frac{n-k}{n}} \quad(*)_{\infty}
$$

for a universal $C_{k, n}^{-}>0$. 


\subsection{Remarks}

(a) One sees easily by looking at particular families of $Y$ 's that the corresponding limsup is bounded by $C_{n k}^{+}(\operatorname{Vol} X)^{\frac{n-k}{n}}$, but it is unclear if $C^{+}=C^{-}$, for all $X$. On the other hand, the Weyl type asymptotic formulas for nonlinear spectra (see $[\text { Gro }]_{\mathrm{DNL}}$ ) suggest a possibility of replacing "lim inf" in $(*)_{\infty}$ by plain "lim".

(b) The inequality $(+)_{d}$ can be seen in the "non-linear spectral framework" as a reflection of semiadditivity of a certain topological colenght (function) on the space $\mathcal{C}_{-k}$ of suitable "codimension $k$ cycles $Y \subset X$ ":

$$
\text { colength } \mathcal{A} \cap \mathcal{B} \leq \text { colength } \mathcal{A}+\text { colength } \mathcal{B}
$$

for all $\mathcal{A}, \mathcal{B} \subset \mathcal{C}_{-k}$. An archetypical example of colength is seen in the projective space $P^{\infty}$ : this is the cohomological length of the complement to $\mathcal{A} \subset P^{\infty}$, i.e. the maximal power $d$ of the generator $h \in H^{1}\left(P^{\infty} ; \mathbb{Z}_{2}\right)=\mathbb{Z}_{2}$ that does not vanish on $P^{\infty} \backslash \mathcal{A}$.

\subsection{Evaluation of waist for specific families $\{Y\}$}

This seems non-trivial even for the simplest examples such as the family of $d$-tuples of $(n-k)$-planes in projective spaces $X$ over $\mathbb{R}, \mathbb{C}$, or $\mathbb{H}$. One knows, however, the minimal $\varepsilon$ for which $Y+\varepsilon=X$ in the $\mathbb{R}$ and $\mathbb{C}$-cases due to theorems of Bang and Ball (see [Ball]). Such an $\varepsilon$ can be essentially sharply evaluated for every algebraic hypersurface $Y \subset \mathbb{R} P^{n}$ of degree $d$ by an appeal to the Bernstein inequality as in Chapter $3 \frac{1}{2}$ of [G-L-P],

$$
\|\operatorname{grad} f\| \leq d \sup \|f\|,
$$

for all polynomials $f: S^{n} \rightarrow \mathbb{R}$ of degree $d$; consequently, the above $\varepsilon$ is $\geq \pi / d$.

Question. Let $Y_{1}, \ldots, Y_{m}$ be algebraic subvarieties in a projective space $X$ of degrees $d_{i}$ and codimension $k_{i}$. What is the range of values of the sum of the volumes of their respective $\varepsilon_{i}$-neighbourhoods for given $\varepsilon_{i}>0$, $i=1, \ldots, m$ ? For which $\varepsilon_{i}$ may these neighbourhoods $Y_{i}+\varepsilon_{i}$ cover all of $X ?$

Let $X$ be a compact symmetric space (e.g. $S^{n}$ ) and $f: X \rightarrow \mathbb{R}^{d}$ be a Veronese map, i.e. $f$ is isometric equivariant (where the implied representation of the isometry group of $X$ may or may be not assumed irreducible). What is the waist of the family $\{Y\}$ in $X$ induced by (intersecting $f(X)$ 
with) $(d-k)$-planes in $\mathbb{R}^{d}$ ? Are the above special families $\{Y\}$ (for standard spaces $X$ ) extremal for the waist or some waist-like invariants?

What is the minimal waist of the family induced on $S^{n}$ from the family of $d$-tuples of points in $\mathbb{R}^{k}$ ?

Let $X \subset S^{N}$ be a semialgebraic subset of dimension $n$ and degree $\delta$. Can one universally bound from below wst $_{-k}^{d}(X)$ for $d \gg \delta$ ? (Here one is tempted to use suitable semialgebraic partitions of $X$, e.g. induced by convex partitions of $S^{N}$, but even the apparently simpler problem of estimating the spectrum of $\Delta$ on $X$ remains open, compare [Gro $]_{\mathrm{SG}}$.)

The above question is better suited for semialgebraic measures in $\mathbb{R}^{N}$ of degree $\leq \delta$. These are derived in the semialgebraic category from the Lebesgue measure $d x$ by the following operations

1. Restricting a measure to a semialgebraic subset (e.g. $d x$ itself on an open semialgebraic $X \subset \mathbb{R}^{N}$ ).

2. Pushforwards under semialgebraic maps.

3. (Canonically) inducing on fibers of semialgebraic maps.

4. Cartesian products of measures and, more generally, integration of semialgebraic families of measures on fibers of semialgebraic maps.

A measure $\mu$ arizing this way can be assigned (negotiably) its degree $\delta=$ $\delta(\mu)$ reflecting (summing up) the (totality of) degrees of the (semi)algebraic operations generating $\mu$. The problem is of evaluating (non-linear) spectral invariants of $\mu$ (waists included) in terms of $\delta(\mu)$ (where the relevant metric may or may be not assumed Euclidean). Also there may be some sense in evaluating the rate of approximation of general measures $\mu$ by semialgebraic $\mu_{d}$ of degrees $d$ for $d \rightarrow \infty$.

Given a polynomial map $f: S^{n} \rightarrow \mathbb{R}^{k \leq n}$ of degree $d$. What is the (best) bound on $\sup \left\|\Lambda^{k} d f\right\|$, i.e. the norm of the differential $d f$ on $k$-vectors, in terms of the $k$-measure of the image $f\left(S^{n}\right) \subset \mathbb{R}^{k}$ (and possibly something like the diameter of the image) in the spirit of Bernstein's inequality?

Estimating volumes of $\varepsilon$-neighbourhoods of algebraic subsets $X$ of codimension $k$ and degree $d$ reduces to the codimension 1 case, if $X$ can be included into a hypersurfaces of degree $d^{\prime}$ much smaller than $d$ as it happens for complete intersections where $d^{\prime}$ is $\leq d^{\frac{1}{k}}$. This does not give much, however, for such $X$ 's as unions of linear subspaces; yet one wonders.

The interest in the study of geometric characteristics of (families of) subsets $Y \subset X$ of given codimension and complexity, (e.g. degree in the (semi)algebraic case) is warmed up by the infinite dimensional examples of 
$X$ 's, such as Grassmannians over Banach spaces and infinite products of $m m$ spaces with a.e. infinite distance.

\section{Isoperimetric recollections (compare [Led])}

If $k=1$, then the geometry of waists imbedds into the classical isoperimetric framework, where one associates, to each space $X=(X$, dist, $\mu)$, its (raw) isoperimetric profile given by a monotone correspondence $v \leftrightarrow s$, that is a subset $I=\left(v_{X}(s), s_{X}(v)\right)$ in $\mathbb{R}_{+}^{2}$ defined as follows. Consider the set (space) $\mathcal{U}$ of all domains $U \subset X$ with $\mu(U)<\infty$ and $\operatorname{Min}_{-1} \partial U<\infty$, where for $\mu(X)<\infty$ one additionally assumes that $\mu(U) \leq \frac{1}{2} \mu(X)$. (Here $\partial U$ denotes the topological boundary if $U$ and $\operatorname{Min}_{-1} \partial U$ stands for the Minkowski measure.) Consider the map from $\mathcal{U}$ to $\mathbb{R}_{+}^{2}$ given by $U \mapsto(v=$ $\left.\mu(U), s=\operatorname{Min}_{-1} \partial U\right)$, let $\mathcal{I} \subset \mathbb{R}_{+}^{2}$ denote the image of this map and define $I$ as the minimal closed subset in $\mathbb{R}_{+}^{2}$, such that for every pair $(v, s) \in \mathcal{I}$ there exists a point $\left(v^{\prime}, s^{\prime}\right) \in I$, where $v^{\prime} \geq v$ and $s^{\prime} \leq s$. If $I$ projects one-toone to the $s$-coordinate, the resulting (monotone increasing) function $v_{X}(s)$ furnishes the (sharp) isoperimetric inequality

$$
\mu(U) \leq v_{X}\left(\operatorname{Min}_{-1} \partial U\right)
$$

for all $U \in \mathcal{U}$; if $I$ has such a projection to $v$ 's, one has $\operatorname{Min}_{-1} \partial U \geq$ $s_{X}(\mu(U))$. In what follows, the both projections are usually (assumed) oneto-one and $I$ equals the common graph of the pair of mutually inverse continuous strictly monotone increasing functions $v_{X}(s)$ and $s_{X}(v)$. Even if this fails to the true, we still write $v(s)$ and $s(v)$, where the monotone functions $v(s)$ and $s(v)$ may become discontinuous and/or non-strictly monotone.

\subsection{Isoperimetry in $\mathbb{R}$ and domination}

Given a measure $\nu$ on $\mathbb{R}$ we regard $(\mathbb{R}, \nu)$ as an $m m$ space and denote its isoperimetric profile $I$ by $\left(v_{\nu}(s), s_{\nu}(v)\right)$. If $f: X \rightarrow \mathbb{R}$ is a 1-Lipschitz map then, clearly, the pushforward measure $\nu=f_{*}(\mu)$ satisfies

$$
\left(v_{\nu}(s) \leq v_{X}(s), s_{\nu}(v) \geq s_{X}(v)\right)
$$

thus the (concentration) behavior of $\nu$ can be seen in the properties of the profile $I$ of $X$.

Conversely, given a domain $U \subset X$, one considers the 1-Lipschitz function $d=d_{U}: X \rightarrow \mathbb{R}$ equal $\operatorname{dist}(x, U)$ for all $x$ outside $U$ and $d(x)=$ 
- $\operatorname{dist}(x, X \backslash U)$ for $x \in U$. The pushforward measure $\nu=d_{*}(\mu)$ on $\mathbb{R}$ encodes the measure of $U$, as $\nu(-\infty, 0]$, and $\operatorname{Min}_{-1} \partial U$, as the density of $\nu$ at zero with respect to Lebesgue measure. Thus the isoperimetric profile $v_{X}(s)$ of $X$ equals the supremum of these for the 1-Lipschitz pushforwards of $\mu$ to $\nu$ on $\mathbb{R}$ and $s_{X}(v)$ equals the corresponding infimum.

Remark. Not every continuous map $f: X \rightarrow \mathbb{R}$ admits a pushforward $f_{*}(\mu)$ : one needs some $\mu$-properness of $f$, e.g. the assumption $\mu\left(f^{-1}[a, b]\right)<$ $\infty$ for all segment $[a, b] \subset \mathbb{R}$ for $-\infty<a \leq b<+\infty$. In what follows, we shall tacitly assume that $f_{*}(\mu)$ is defined whenever this is needed.

A measure $\nu$ on $\mathbb{R}$ is called iso(perimetrically)-simple if its support is connected and its isoperimetric profile equals the closure of the pairs of points $\left(v=\mu(-\infty, v), s=\operatorname{Min}_{-1}\{t\}\right)$, where $\operatorname{Min}_{-1}=d \mu / d t<\infty$. In other words, the subsets $(-\infty, t] \subset \mathbb{R}$ solve the isoperimetric problem for $(\mathbb{R}, \nu)$.

9.1.A Lemma (Isoperimetric inequality in $\mathbb{R}$ ). Let $\nu$ be given by a strictly positive continuous density function, $\nu=\varphi(t) d t$ on some open, finite or infinite, segment in $\mathbb{R}$. Then $\nu$ is iso-simple in the following two cases.

1. $\varphi(t)$ is strictly monotone increasing.

2. $\mu(X)<\infty$, the function $\varphi$ is even $(\varphi(t)=\varphi(-t))$, strictly monotone increasing for $t<0$ and the corresponding function $s_{\nu}(v)$ is sublinear for $v \leq \frac{1}{2} \mu(X)$,

$$
s_{\nu}\left(v_{1}+v_{2}\right) \leq s_{\nu}\left(v_{1}\right)+s_{\nu}\left(v_{2}\right), v_{1}+v_{2} \leq \frac{1}{2} \mu(X) .
$$

This is straightforward, as well as the following

9.1.B Domination corollary. Let $\nu$ satisfy 1 or 2 and let $f: \mathbb{R} \rightarrow \mathbb{R}$ be a 1-Lipschitz map that is assumed $\mu$-proper in the case 1 . Then there exists a monotone increasing 1-Lipschitz function $\underset{\rightarrow}{f: \mathbb{R}} \rightarrow \mathbb{R}$, unique on the segment where $\varphi>0$, such that $\underset{*}{f_{*}}(\nu)=f_{*}(\nu)$.

9.1.C Iso-domination. A measure $\nu$ on $\mathbb{R}$ (preferably but necessarily isosimple) is called an iso-dominant of $X=(X$, dist, $\mu)$ if for every $\mu$-proper Lipschitz map $f: X \rightarrow \mathbb{R}$ there exists a monotone increasing 1-Lipschitz

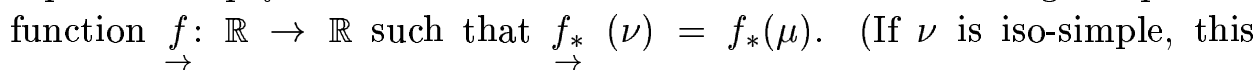
is equivalent to $\left(v_{\nu}(s) \geq v_{x}(s), \overrightarrow{s_{\nu}}(v) \leq s_{X}(v)\right)$.) An iso-dominant is called 
minimal if it is iso-dominated by the other iso-dominants of $X$. (If $v_{X}(s)$ and $s_{X}(v)$ are honest functions, there is a unique minimal dominant $\nu=\varphi(t) d t$ where $\varphi$ solves the differential equation $(\log \varphi)^{\prime}=v^{\prime}(\varphi)$.)

If we drop the monotonicity assumption on $\underset{f}{f}$, we arrive at a more general notion: (non-iso)domination of $X$ by $\nu$. If $\vec{\nu}$ is iso-simple, then, clearly, domination $\Rightarrow$ iso-domination.

An (iso)-dominant $\nu$ is called sharp, if it equals the pushforward of $\mu$ under a 1-Lipschitz function $f: X \rightarrow \mathbb{R}$ that is called (iso)-extremal: the levels of $f$ solve the isoperimetric problem in $X$.

Remarks. (a) If the isoperimetric profile $s_{X}(v)$ is sublinear, the isoperimetric inequality for disconnected domains $U \subset X$ reduces to that for connected ones. Otherwise, one should keep track of individual connected components of $U$, possibly, by introducing a counterpart to iso-domination for map of $X$ not to $\mathbb{R}$, but rather to certain 1-dimensional spaces (trees or more general graphs), where the pull-backs of the points are connected.

(b) A special feature of $\mathbb{R}$ is the existence of unique monotone decreasing function pushing forward a (standard) measure $\nu$ to a given $\nu^{\prime}$ under obvious assumptions that are easily satisfied. This is not so in higher dimensional spaces $Z$ where neither existence of such a, say dominating 1-Lipschitz map $Z \rightarrow Z$, nor its uniqueness are available. For example, if $f^{\prime}: X^{\prime} \rightarrow \mathbb{R}^{k}$ is a generic smooth map and $\operatorname{dim} X^{\prime} \geq k+2$, then the density of the measure $\nu^{\prime}=f_{*}^{\prime}\left(\mu^{\prime}\right)$ vanishes on the boundary $\partial^{\prime}$ of the image $f^{\prime}\left(X^{\prime}\right) \subset \mathbb{R}^{k}$. Thus every measure $\nu$ on $\mathbb{R}^{k}$ dominating $\nu^{\prime}$ needs to have a vanishing density locus (hypersurface) $\partial_{0}$ admitting a 1-Lipschitz map onto $\partial^{\prime}$ : this is rather hard on $\nu=f_{*}(\mu)$.

(c) One can sometimes regain the uniqueness of a measure preserving map $\underset{\rightarrow}{f}$ for $\operatorname{dim} \geq 2$ by requiring extra properties of this $\underset{\rightarrow}{f}$. For example, let $Z$ comes as the (final total) space of a power of $\mathbb{R}$-fibrations

$$
Z \stackrel{\mathbb{R}}{\rightarrow} Z_{-1} \stackrel{\mathbb{R}}{\rightarrow} \cdots \rightarrow Z_{-k+1}=\mathbb{R}
$$

(e.g. a simply connected nilpotent Lie group $Z$ decomposed into a sequence of central $\mathbb{R}$-extensions). Suppose we have a subset $U \subset Z$ such that the projection $U_{-i} \subset Z_{-i}, i=0,1, \ldots, k-1$, of $U$ to each $Z_{-i}$ has connected intersection with the $\mathbb{R}$-fibers of the following projection $U_{-i} \rightarrow Z_{-i-1}$. Then, given an arbitrary $U$ with $\mu(U)=\mu\left(U^{\prime}\right)$, for a given smooth measure $\mu$ on $Z$, there (obviously) exists a unique (Knöthe) map $U^{\prime} \rightarrow U$ preserving $\mu$ and compatible with the $\mathbb{R}$-tower structure: each intersection $U_{-i}^{\prime} \cap \mathbb{R}_{z^{\prime}}$, 
$z^{\prime} \in Z_{-i-1}$, is sent to $\mathbb{R}_{z} \subset U_{z}$ by a monotone map, for all $i=0,1, \ldots, k-1$. (Such a map for $\mathbb{R}^{n} \rightarrow \mathbb{R}^{n-1} \rightarrow \cdots \rightarrow \mathbb{R}$, instantaneously leads to a proof of the isoperimetric inequality and Brunn-Minkowski inequality in $\mathbb{R}^{n}$, see [Mil-Sch]).

One can envizage other prefered choices of (not necessarily equidimensional) "measure parametrization", e.g. by Gaussian spaces or by Kähler manifolds (via moment maps as in [Gro $]_{\text {CSKM }}$ ), where one may also use $f$ 's minimizing a suitable (transportation) energy (in the spirit of Brenier map, see [Gia-Mil]).

\subsection{Standard inequalities}

Take an arbitrary probability measure $\mu$ on the sphere $S^{n}$, say with a positive bounded density, and consider all convexly derived measures $\mu^{\prime}$ on the minimal geodesic segments $S \subset S^{n}$. Let dist be a metric on $S^{n}$, take its restrictions to the segments $S$ and define $(\bar{v}(s), \underline{s}(v))$ by

$$
\bar{v}(s)=\sup v(s), \underline{s}(v)=\inf s(v)
$$

where the sup and inf are taken over the isoperimetric profiles of all ( $S$, dist | $S, \mu^{\prime}$ ) (where $\left(S\right.$, dist $\mid S$ ) may come with many probability measures $\mu^{\prime}$ corresponding to various weak limits of normalized $\mu \mid U_{i}$, for convex domains $U_{i}$ converging to a given $S$ ).

9.2.A. (compare [Gro-Mil]) The isoperimetric profile of $X=\left(S^{n}\right.$, dist, $\left.\mu\right)$ satisfies

$$
v_{X}(s) \leq \bar{v}(s), s_{X}(v) \geq \underline{s}(v) .
$$

Proof. (compare [Gro-Mil]) Take an arbitrary $U \subset S^{n}$ and observe that there obviously exists a convex partition of $S^{n}$ into geodesic segments $S$, such that the canonical probability measure $\mu_{S}$ on $S$ satisfies, for almost all $S$

$$
\mu_{S}(S \cap U)=\mu(U) .
$$

Each $\mu_{S}$ can be convexly derived from $\mu$ and thus the isoperimetric inequalities on $S$ 's integrate to that on $S^{n}$.

9.2.B Corollary. (Euclid?) Spherical isoperimetric inequality. The distance function to a point $s_{0} \in S$, that is $f(s)=\operatorname{dist}\left(s, s_{0}\right)$ is iso-extremal for the standard geometry on $S^{n}$. Furthermore, this remains valid for every convex sector, i.e. a subset $X \subset S^{n}$ containing $s_{0}$ and $-s_{0}$. 
Proof. The convexly derived measures on the segments are $\sin ^{n-1}$-concave and hence are iso-dominated by (properly normalized) (iso-simple!) measure $\sin ^{n-1} t d t$ on $[0, \pi]$.

9.2.C Euclidean subcorollary. Let $X \subset \mathbb{R}^{n}$ be a convex cone. Then the distance to the origin, $f(x)=\|x\|$, is iso-extremal on $X$.

Proof. Go to the limit, $\mathbb{R}^{n}=\lim _{\lambda \rightarrow \infty} \lambda S^{n}$.

Questions. (a) Is there similar scaling limit bringing the waist inequality from (sectors in) $S^{n}$ to $\mathbb{R}^{n}$.

(b) The above is also true for "slightly concave" cones by the stability of the implied (variational) boundary value problem, but exact condition is unclear. It is worthwhile to consider (warped) cones $X$ over arbitrary Riemannian manifolds (and $m m$-spaces) and identify those where $\operatorname{dist}\left(x_{0}, x\right): X \rightarrow \mathbb{R}$ is iso-extremal for the apices $x_{0}$ of the cones $X$.

(c) The partition proof of the isometric inequality for hypersurfaces $Y$ in $\mathbb{R}^{n}$ and $S^{n}$ suggests the following refinement of Almgren's filling isoperimetric inequality. Let $Y \subset \mathbb{R}^{N}$ (or $Y \subset S^{N}$ ) be an $(n-1)$ )-dimensional cycle, e.g. a closed (piece-wise) smooth manifold with $\operatorname{Vol}_{n-1} Y \leq \operatorname{Vol} S^{n-1}$. One seeks a ruled filling $U$ of $Y$ partitioned into straight (geodesic in $S^{N}$ ) segments of length $\leq 1$, such that a thin tube $T$ around almost every segment reaches the boundary $Y$ in $U$ and has $n$-volume $\leq \frac{1}{n} \operatorname{Vol}_{n-1}(T \cap Y$ ) (with the obvious modification for $Y \subset S^{N}$ ).

(d) Let $X$ be an irreducible symmetric space of non-compact type. Does it admit an iso-extremal function $f: X \rightarrow \mathbb{R}$ ? If so, it seems easy to show that such an $f$ is invariant under the isotropy subgroup $G_{0}$ of a point $x_{0} \in X$ and then its identification reduces to the corresponding isoperimetric problem for the Weyl chamber $X / G_{0}$ with the pushforward of the Riemannian measure of $X$ to $X / G_{0}$. In particular, if $\mathbb{R}$-rank $X=1$, then one (naively?) expects that $f(x)=\operatorname{dist}\left(x_{0}, x\right)$ is iso-extremal.

(e) Let $X=\mathbb{R}^{n}$ with $0(n)$-invariant distance and measure. When is the distance function to the origin, $f(x)=\operatorname{dist}(x, 0)$ iso-extremal? Here is a standard counter example, where alternative iso-extremal functions exist.

9.2.D Borel's Gaussian isoperimetric inequality. Normal projections of $\mathrm{Ga}^{n}=\left(\mathbb{R}^{n}, e^{-\|x\|^{2}} d x\right)$ to lines in $\mathbb{R}^{n}$ are iso-extremal. 
Proof. The derived measures of $e^{-\|x\|^{2}} d x$ are 2-log-concave and, hence, iso-dominated by $e^{-t^{2}} d t$ (with all measures being normalized).

\subsection{Paul Levy inequality}

One can productively apply 9.2.A to metrics dist associated to the projective structure in $S^{n}$ see [Gro-Mil] but, in general, one would prefer dist-geodesics rather than the spherical ones. Here one needs the following modifications of convex derivation of measures.

9.3.A Normal geodesic partition. A partition of a metric space $X$ into topological segments $S$ is called normal geodesic if there exists a 1-Lipschitz function $d: X \rightarrow \mathbb{R}$ that is isometric on each segment $S$. If $\mathcal{C}$ is a concavity class of measures $\varphi(t) d t$ on $\mathbb{R}$, we say that $X=(X$, dist $\mu)$ is $\mathcal{C}$-concave if the canonical measures on almost all $S$, (when transported to $\mathbb{R}$ by $d$ ) are in this class for all normal geodesic partitions. If $X$ is a smooth manifold, it is called smoothly $\mathcal{C}$-concave, if this holds for partitions admitting an almost everywhere smooth function $d$. (This is an apparently weaker, but probably, an equivalent condition for smooth $X$.)

Levy domination inequality. Let $X=(X$, dist, $\mu)$ be a compact smooth Riemannian pm space, where $X$ may have non-empty convex boundary and where smoothness of $\mu$ means that $\mu=\varphi(x) d x$ for $\varphi(x)>0$ and $C^{2}$-smooth. If $X$ is smoothly $\sin ^{\alpha}$-concave for some $\alpha>0$ then it is iso-dominated by the normalized measure $\sin ^{\alpha} t d t$ on $[0, \pi]$.

This is proved in [G-L-P] for $\alpha=\operatorname{dim} X-1$ and $\mu$ being the normalized Riemannian measure. (This concavity condition is equivalent to $\operatorname{Ricci}(X) \geq n-1=\operatorname{Ricci} S^{n}$.) The (variational) proof (see [G-L-P]) allows an introduction of non-Riemannian $\mu$.

Remarks and questions. (a) The above seems to work for some (all?) Finsler manifolds $X$ and, possibly, one can completely drop the smoothness assumption.

(b) Similar (domination) inequalities can be proved, at least in the smooth case, for other $\mathcal{C}$ 's, e.g. for 1 -log-concave $X^{\prime}$, that are easily seen to be iso-dominated by $e^{-t^{2}} d t$, but I have not looked for meaningful examples.

(c) Let $X$ be an $n$-dimensional Riemannian manifold with sectional curvature $K(X) \geq 1$ (or an Alexandrov's space with $K \geq 1$ ). Does it have the codimension $k$ waist $\geq \mu\left(S^{n-k}+\varepsilon\right)$ for $S^{n-k} \subset S^{n}$ (where the measures 
in question are normalized Riemannian)? What are possible (plausable) non-Riemannian measures in this context?

(d) Can one replace the variational method of Levy by a (topological) partition argument? Ideally, given $U \subset X$, one would like partition $X$ into geodesic segments $S$, such that $\mu_{S}(U \cap S)=\mu(U)$ for all $S$ for the canonical measures $\mu_{S}$, where, moreover, this partition is normal; but this does not seem realistic.

(e) Given two "geometric" measure spaces $A$ and $A^{\prime}$ are routinly proves (isoperimetric) inequalities of the form $\mu(A) \leq I\left(\mu^{\prime}\left(A^{\prime}\right)\right)$ for a given function $I(t)$ by partitioning (or rearranging) the spaces, and then integrating over the partitions (integral geometry, symmetrization etc.). In the present paper the relevant partition $\Pi$ emerges as a solution of a certain system of (infinitely many) functional equations, where the solvability of these is derived from their homological non-triviality. Can one extend the topological method to other situations where the present (rearrangment) proofs rely on P.D.E., such as the Alexandrov-Fenchel inequality? The analytic proof of Alexandrov is suggestive as it depends on "complex rearrangment" of the relevant integral via some linear Hodge operator of zero index, i.e. with non-zero topological degree (see [Gro] $]_{\mathrm{CS}}$ ). Similar, but non-linear "complex rearrangments" can be seen in the proofs of inequalities between Chern numbers of stable (tangent) bundles over (of) Kähler manifolds (Bogomolov-YauAubin-Simpson...). Can one see this topologically and trace the topology to analysis and/or the algebraic geometry of the manifolds in question?

(f) Is there a calculus of variations in the spaces of (convex) partitions? If so, is it compatible with the Gibbsian geometry in the space of micells indicated in $[\mathrm{Ca}-\mathrm{Gr}]$ ?

\subsection{Symmetrization and isoperimetry of products}

Let $X$ be partitioned into closed subsets $X_{b}$ that are the fibers of a continuous map $p: X \rightarrow B$ and concentrate on the isoperimetric problem over $B$, where one looks for a subset (domain) $U \subset X$ with prescribed measures $\mu_{b}\left(U \cap X_{b}\right), b \in B$ having minimal Min $_{-1} \partial U$, where $\mu_{b}$ denote the canonical measures in the fibers $X_{b}=p^{-1}(b)$. In other words, one prescribes a function $v$ on $B$ and minimizes $\operatorname{Min}_{-1} \partial B$ over $U$ 's with $p_{*}(\mu \mid U)=v p_{*}(\mu)$ (with an obvious interpretation/modification for non- $\mu$-proper $p$ ).

Let $p^{\prime}: X^{\prime} \rightarrow B$ be another $m m$ space over $B$ where the fibers are oriented topological lines $\mathbb{R}_{b}=\mathbb{R}$, such that the canonical measures $\mu_{b}^{\prime}$ are

finite on $(-\infty, t] \in \mathbb{R}_{b}=\left(p^{\prime}\right)^{-1}(b)$ for all $t<\infty$ and $b \in B$. Assume 
the canonical measures $\mu_{b}^{\prime}$ on $\mathbb{R}_{b}$ 's have no atoms and $\mu_{b}^{\prime}\left(\mathbb{R}_{b}\right) \geq \mu_{b}\left(X_{b}\right)$ for all $b \in B$. Then for each $U \subset X$ there exists a unique $U^{\prime} \subset X^{\prime}$, such that $U^{\prime} \cap \mathbb{R}_{b}=\left(-\infty, t_{b}\right]$ with $\mu^{\prime}\left(-\infty, t_{b}\right)=v_{b}$, called the (Schwarz) symmetrization of $U$.

9.4.A. Let us compare the Minkowski measure of the "hypersurface" $H=$ $\partial U$ with those of the intersections $H_{b}=H \cap X_{b} \subset X$. Let $d=d_{H}(x)=$ $\operatorname{dist}_{X}(x, H)$ and $D=D_{H}(x)=\operatorname{dist}_{X_{b}}\left(x, H_{b}\right)$ for $b=p(X)$. Clearly $d<D$ and the ratio $\Delta=\Delta_{H}=D / d$ represents the "slope" of $H$. Say that (a closed subset $H \subset X \rightarrow B$ ) is regular, if the function $\Delta_{H}$ continuously extends from the complement $X \backslash H$ to $H$.

Call $U$ strongly regular, if $\partial U$ is a.e. regular and

$$
\operatorname{Min}_{-1} \partial U=\int_{B} d b \int_{H_{b}} \Delta_{H}(h) d h_{b}
$$

where $d h_{b}$ refers to the Minkowski measure in $H_{b} \subset X_{b}$ that is assumed good enough to use for integration.

Say that $X^{\prime}$ iso-restricts $X$ if $\operatorname{Min}_{-1} \partial U^{\prime} \in \operatorname{Min}_{-1} \partial U$ for all those strongly regular $U$, where $U^{\prime}$ is also strongly regular.

Since $\mu^{\prime}\left(U^{\prime}\right)=\mu(U)$, this makes the isoperimetric inequality in $X^{\prime}$ stronger than that in $X^{\prime}$,

$$
v_{X}(s) \leq v_{X^{\prime}}(s), s_{X}(v) \geq s_{X^{\prime}}(v) .
$$

Furthermore, if the spaces $X$ and $X^{\prime}$ themselves are sufficiently regular, e.g. smooth Riemannian with smooth measures and with the maps $X, X^{\prime} \rightarrow$ $B$ being smooth submersions, then the isoperimetric inequality for strongly regular domains $U$ implies that for all $U$ and one should not bother much about strong regularity. Call $H$ horizontal at a point $h \in H$, if $\Delta_{H}(h)=1$.

Now, turn to $p^{\prime}: X^{\prime} \rightarrow B$, assume it is split into the product, $X^{\prime}=B \times \mathbb{R}$, and postulate the following normality of this splitting: a regular graph $H^{\prime} \subset$ $X$ of a function $\sigma: B \rightarrow \mathbb{R}$ is horizontal at a point $h^{\prime}=\sigma(b) \in H^{\prime}$ if and only if $\nabla^{\prime} \sigma(b)=0$, that is $\sigma(b)-\sigma\left(b^{\prime}\right)=o\left(\operatorname{dist}_{X^{\prime}}\left(\sigma(b), \sigma\left(b^{\prime}\right)\right)\right.$ for $b^{\prime} \rightarrow b$.

The next symmetrization postulate concerns $X$ : if the boundary $\partial U$ is horizontal at all point $h \in p^{-1}(b) \cap \partial U$ for some $b_{0} \in B$ then the function $\sigma_{U}: b \mapsto \mu_{b}\left(U_{b}\right)$ has $\nabla^{\prime} \sigma_{U}\left(b_{0}\right)=0$ for all $U \subset X$ with regular boundaries. (Observe, this depends on the metric dist' assigned to $X^{\prime}=X \times \mathbb{R}$.)

This implies together with the previous postulate that the symmetrization $U \mapsto U^{\prime}$ preserves horizontality of the boundaries over all points $b \in B$.

Coming back to $X^{\prime}=B \times \mathbb{R}$ we strengthen the first postulate by requiring the existence of a continuous function $\varphi^{\prime}=\varphi^{\prime}(\delta, b), \delta \in \mathbb{R}_{+}, b \in B$, monotone 
increasing in $\delta$, such that $\Delta_{H^{\prime}}(\sigma(b)) \leq \varphi^{\prime}\left(\nabla^{\prime} \sigma(b), b\right)$ for all functions $\sigma: B \rightarrow$ $\mathbb{R}$ with regular graphs $H^{\prime} \subset X^{\prime}=B \times \mathbb{R}$ and almost all $b \in B$.

Example. If $X^{\prime}$ is a Riemannian product, $X^{\prime}=B \times \mathbb{R}$, then $\Delta=\sqrt{1+\nabla^{2}}$ and $\varphi^{\prime}(\delta)=\sqrt{1+\delta^{2}}$ does the job.

Finally, we require that there is a continuous function $\psi(\theta, b)$ monotone increasing in $\theta$ such that

$$
\operatorname{Av}_{H_{b}} \psi\left(\Delta_{H}(h)\right) \geq\left(\nabla^{\prime} \sigma_{U}(b)\right) / \sigma_{U}(b)
$$

for all $U$ with regular boundaries, where the average "Av" is taken with respect to the Minkowski measure in $H_{b} \subset X_{b}$ under the assumption this is well defined; furthermore, we require that the inverse function $\varphi(\delta, v)$ to $\psi(\theta, v)$ (i.e. $\varphi(\psi(\theta, v), v)=\theta)$ majorizes the above $\varphi^{\prime}(\delta, v)$.

Example. If $X=B \times X$ • is a Riemannian product, then the (obvious) $\psi$ is $\psi(\theta, v)=\sqrt{\theta^{2}-1}$.

9.4.B Schwarz symmetrization lemma. Granted the assumptions above, the space $X \rightarrow B$ is iso-restricted by $X^{\prime}=B \times \mathbb{R}$, provided the function $\varphi(\theta, v)$ is convex in $\theta$.

Proof. Follow through the above definitions observing that

$$
\operatorname{Av}_{H_{b}} \Delta(h) \geq \varphi(\operatorname{Av} \psi(\Delta(h)))
$$

for convex functions $\varphi=\varphi(\delta)$ and their inverses $\psi$.

Schwarz' lemma may be applied twice: first to $X \rightarrow B$ and then to $X^{\prime}=B \times \mathbb{R} \rightarrow \mathbb{R}$. Thus isoperimetric inequality for $X$ reduces to that for the plane $X^{\prime \prime}=\left(\mathbb{R} \rightarrow \mathbb{R}, \nu^{\prime} \times \nu^{\prime \prime}\right)$, where, moreover one only needs to check the inequality for monotone domains $U^{\prime \prime} \subset X$, where the intersections of $U^{\prime \prime}$ with the (vertical and horizontal) lines $t \times \mathbb{R}$ and $\mathbb{R}=\mathbb{R} \times s$ are segments of the form $(-\infty, t]$.

The essential condition for applying the lemma is the convexity of $\varphi(\delta)$, that is available for a rather wide class of fibration of (mildly) singular Finsler manifolds. The classical example is the following

9.4.C Schwarz' product theorem. Let $X_{i}=\left(X_{i}, \operatorname{dist}_{i}, \mu_{i}\right), i=1, \ldots, r$, be Riemannian manifolds with smooth measures $\mu_{i}$ iso-dominated by measures $\nu_{i}$ on $\mathbb{R}$. Then the isoperimetric profile $\left(v_{X}, s_{X}\right)$ of $X=X_{1} \times \cdots \times X_{r}$ is restricted by that of $X^{\prime}=\left(\mathbb{R}, \nu_{1}\right) \times \cdots \times\left(\mathbb{R}, \nu_{r}\right)$,

$$
v_{X}(s) \leq v_{X^{\prime}}(s), s_{X}(v) \geq s_{X^{\prime}}(v) .
$$


Indeed, the function $\sqrt{1+\delta^{2}}$ is convex and Schwarz' lemma applies.

9.4.D. Generalization to fibrations. Let us enumerate the essential features of the products, say for $r=2$, that are needed to apply Schwarz' lemma and that allow an extension of Schwarz' theorem to fibration $p: X \rightarrow$ $\underline{X}$.

1. The measures $\mu$ on $X, \underline{\mu}$ on $\underline{X}$ and $\mu_{\underline{x}}$ in the fibers $X_{\underline{x}}=p^{-1}(\underline{x})$, $\underline{x} \in \underline{X}$, must agree in the obvious manner:

$$
\int_{X} \psi(x) d \mu=\int_{\underline{X}} d \underline{\mu} \int_{X_{\underline{x}}} \psi(\underline{x}) d \mu_{\underline{x}}
$$

for all continuous functions $\psi$ on $X$ with compact supports.

2 . The measures $\mu_{\underline{x}}$ are invariant under the parallel transport normal to the fibers. This means that the map $p: X \rightarrow \underline{X}$ is a smooth submersion and every local vector field in $X$ normal to the fibers and moving fibers to fibers preserves the measures $\mu_{\underline{x}}$.

3. The map $p: X \rightarrow \underline{X}$ is 1-co-Lipschitz: the Hausdorff distance between every two fibers satisfies, $\operatorname{dist}_{\mathrm{Hau}}\left(X_{\underline{x}_{1}}, X_{\underline{x}_{2}}\right) \leq \operatorname{dist}_{\underline{X}}\left(\underline{x}_{1}, \underline{x}_{2}\right)$.

Granted 1, 2 and 3 one has

Schwarz fibration theorem. If $\underline{X}$ is iso-dominated by $\nu_{1}$ and all fibers $X_{\underline{x}}$ by $\nu_{2}$, then the isoperimetric profile of $X$ is restricted by that of $X^{\prime}=$ $\left(\mathbb{R}, \nu_{1}\right) \times\left(\mathbb{R}, \nu_{2}\right)$.

This theorem applies, for example, to Riemannian fibrations as well as to warped products. (In the latter case the fiber measures must be normalized with a suitable warping function $\varphi(\underline{x})$.)

Remark. A similar theorem holds true for suitably "infinitesimally split" fibrations of Finsler manifolds.

9.4.E Isoperimetric problems in the plane. Let us write down the Euler-Lagrange (variational) equation for smooth hypersurfaces $H \subset X=$ $(X$, dist, $\mu(x)=\varphi(x) d x)$ with prescribed $\mu(\Omega)$ and having minimal $\operatorname{Min}_{-1} H$. First define mean $\mu$-curvature of $H$,

$$
M_{\mu}(h)=M(h)+\varphi^{-1}(h)\langle\vec{n}(h), \operatorname{grad} \varphi(h)\rangle,
$$

where $M(h)$ is the ordinary mean curvature of $H$ at $h \in H$ and $\vec{n}$ is the unit normal (say inward looking) vector, and where $\varphi(x)$ is assumed smooth and strictly positive in the interior of $X$. 
The Euler-Lagrange equation reads: $M_{\mu}(h) \equiv$ const; moreover $H$ is normal to the boundary $\partial X$ (at least) at those points where $\varphi(x) \neq 0$.

This P.D.E. reduces to an O.D.E. for $X=\left(\mathbb{R}, \nu_{1}\right), x\left(\mathbb{R}, \nu_{2}\right)$ and, whenever solvable, can be used in conjunction with Schwarz' theorem.

Examples. (a) Let $X=\left(\mathbb{R}_{+}^{2}, c_{n} t^{n-1} d t d t^{\prime}\right)$. If $c_{n}=\left(\operatorname{Vol} B(1) \subset \mathbb{R}^{n}\right)$, one can easily show that the relevant solutions are concentric (quarters of) circles and that consequently, $X$ is iso-dominated by the measure $c_{n+1} t^{n} d t$ on $\mathbb{R}_{+}$. This obvious induction on $n$, constitute Schwarzian proof of the isoperimetric inequality in $\mathbb{R}^{n}$.

Remark. One does not actually need to solve the equation $M_{\mu} \equiv$ const for $n \geq 3$, as the symmetries of the ambient space force the solution to be circular (while the case of $n=2$ does need the identification of circles as the only curves of constant curvature).

(b) The Gaussian space $\mathrm{Ga}^{n}$ splits into product of Ga's and Schwarz' theorem yields the Gaussian isoperimetry.

(c) The hyperbolic space $H^{n}$ splits into the warped product $\mathbb{R}_{+} \times{ }_{\varphi} S^{n-1}$ for $\varphi=\varphi(t)=\sin h(t)$. If $n=2$, one accepts the circles on the only curves of constant curvature and the same holds for the planes iso-restricting $H^{n}$ for $n \geq 3$; here circularity of the solutions of the equation $M_{\mu} \equiv$ const follows by the symmetry (in $H^{n}$ for $n \geq 3$ ) argument. As a bonus one solves the isoperimetric problems in convex cones $X \subset H^{n}$ : the distance function to the apex is iso-extremal in every such $X$ (including those with apices at the ideal boundary $\partial H^{n}$ ). Moreover, the same holds true for the warped product $\mathbb{R}_{+} \times_{\varphi(t)} X_{0}$ with $X_{0}$ being an $n$-dimensional Riemannian manifold with $\operatorname{Ricci}\left(X_{0}\right) \geq n-1$ and $\varphi(t)=\sin h t$.

Remarks. (a) Explicit evaluation of the isoperimetric profiles of (warped) products of lines, $\left(\mathbb{R}, \nu_{1}\right) \times\left(\mathbb{R}, \nu_{2}\right)$, may become painful even if the corresponding O.D.E. is readily solvable; this is partly due to a possible discrepancy between domination and iso-domination. Apparently, this is the reason why one still does not know the profiles of product of (round) spheres and of regular simplices (in $S^{n}, \mathbb{R}^{n}$ and $H^{n}$ ), for example. (A more difficult question concerns evaluation of waists of these spaces.)

(b) Schwarz' fibration argument also applies to Minkowski sums and yields Minkowski's (type) inequalities for group extensions, e.g. the ordinary Brunn-Minkowski for the simply connected nilpotent Lie groups. 


\section{Bibliography}

[Ball] Ball, K.M., Complex plank problem, Bulletin of the LMS 33, pp. 433442.

[Bez-Con] Bezdek, K., Connelly, R., Pushing disks apart - The Kneser-Poulsen conjecture in the plane, Preprint (2001).

[Ca-Gr] Carbone, A., Gromov, M., Mathematical slices of molecular biology, Numéro Spécial de la Gazette des Mathématiciens 88:11-80, Société Mathématique de France (2001).

[Gia-Mil] Giannopoulos, A., Milman, V., Euclidean structure in finite dimensional spaces, Handbook of the Geometry of Banach Spaces (JohnsonLindenstrauss eds.), Vol. 1 (2001), pp. 707-779.

[G-L-P] Gromov, M., Metric Structures for Riemannian and Non-Riemannian Spaces, based on: Structures Métriques des Variétés Riemanniennes (with Appendices by M. Katz, P. Pansu, and S. Semmes), edited by J. Lafontaine and P. Pansu, English Translation by Sean M. Bates, Birkhaüser, Boston - Basel - Berlin (1999).

[Gro $]_{\mathrm{CS}}$ Gromov, M., CAT $(\kappa)$-space: construction and concentration, to Victor Abramovich Zalgaller, Preprint IHES, January 2001.

[Gro] CSKM Gromov, M. Convex sets and Kähler manifolds, in Advances in J. Differential Geom., F. Tricerri ed., World Sci., Singapore, (1990), pp. 138.

[Gro]DNL Gromov, M., Dimension, non-linear spectra and width, Lect. Notes in Math., Springer-Verlag 1317 (1988), pp. 132-185.

[Gro $]_{\text {FRM }}$ Gromov, M., Filling Riemannian manifolds, J. Differential Geom. 18 (1983), pp. 1-147.

[Gro $]_{\mathrm{SG}}$ Gromov, M., Spectral geometry of semi-algebraic sets, Ann. Inst. Fourier 42 (1992), pp. 249-274.

[Gro $]_{\text {SI }}$ Gromov, M., Systoles and intersystolic inequalities, Séminaires et Congrès Soc. Math. France 1 (1996), pp. 291-362.

[Gro $]_{\mathrm{SQ}}$ Gromov, M., Spaces and questions, GAFA, Geom. Funct. Anal., Special Volume (2000), 118-161. 
[Gro-Mil] Gromov, M., Milman, V.D., Generalization of the spherical isoperimetric inequality to uniformly convex Banach spaces, Compositio Math. 62 (1987), no. 3, pp. 263-282.

[Led] Ledoux, M., The Concentration of Measure Phenomenon, Mathematical Surveys and Monographs, Volume 89, American Mathematical Society (2001).

[Mil-Sch] Milman, V.D., Schechtman, G., Asymptotic theory of finite dimensional normed spaces, Lecture Notes in Math. 1200 (1986), Springer. 